\title{
Halo streams in the solar neighborhood
}

\author{
Rainer J. Klement
}

Received: 27 May 2010 / Published online: 30 July 2010

(c) The Author(s) 2010. This article is published with open access at Springerlink.com

\begin{abstract}
The phase-space structure of our Galaxy holds the key to understand and reconstruct its formation. The $\Lambda \mathrm{CDM}$ model predicts a richly structured phase-space distribution of dark matter and (halo) stars, consisting of streams of particles torn from their progenitors during the process of hierarchical merging. While such streams quickly loose their spatial coherence in the process of phase mixing, the individual stars keep their common origin imprinted into their kinematic and chemical properties, allowing the recovery of the Galaxy's individual "building blocks". The field of Galactic Archeology has witnessed a dramatic boost over the last decade, thanks to the increasing quality and size of available data sets. This is especially true for the solar neighborhood, a volume of 1-2 kpc around the sun, where large scale surveys like SDSS/SEGUE continue to reveal the full 6D phase-space information of thousands of halo stars. In this review, I summarize the discoveries of stellar halo streams made so far and give a theoretical overview over the search strategies imployed. This article is intended as an introduction to researchers new to the field, but also as a reference illustrating the achievements made so far. I conclude that disentangling the individual fragments from which the Milky Way was built requires more precise data that will ultimately be delivered by the Gaia mission.
\end{abstract}

Keywords Milky Way, dynamics · Milky Way, kinematics · Milky Way, solar neighborhood

\section{Introduction}

Not until the past two decades have we begun to understand the composition of our Galaxy in an universal context of galaxy formation and evolution, thanks to great

\section{R. J. Klement $(\bowtie)$}

Max-Planck-Institut für Astronomie, Königstuhl 17, 69117 Heidelberg, Germany

e-mail:klement@mpia.de 
progress in both observations and theoretical work. Historically, there have been two different models for the early formation of the Milky Way's stellar halo. Eggen et al. (1962) found strong correlations between the metallicity and eccentricity of stars in the Solar neighborhood. To explain the highly eccentric orbits of the oldest stars, they argued that at the time of formation of these stars, the Galaxy could not have been in dynamical equilibrium, but in a state of rapid gravitational collapse $\left(\sim 10^{8}\right.$ year $)$ from a larger homogeneous spheroid, the "protogalaxy". After the Galaxy reached dynamical equilibrium and became rotationally supported, further star formation would have taken place in a metal-enriched disk, thereby explaining the disk-like orbits of the metal-rich stars. This scenario is called a monolithic collapse. Although the correlation between eccentricity and metallicity found by Eggen et al. (1962) is empirically correct for samples including both disk and halo stars, many authors later questioned its validity for a pure halo sample and it was definitively discarded from a kinematically unbiased sample of 1,203 metal-poor stars (Chiba and Beers 2000, and references therein).

In 1978, Searle and Zinn published their seminal study on the distribution of globular clusters in the outer Milky Way halo; they found no abundance gradient, but a substantial spread in the ages of the clusters. They concluded that, although the lack of an abundance gradient would still be consistent with cluster formation during the rapid free-falling phase of a galactic collapse (because the freefall timescale is much shorter than the enrichment timescale), globular clusters would not have such a high internal chemical homogeneity, if they would have formed during a monolithic collapse. Therefore, Searle and Zinn (1978) proposed a slow ( $\gtrsim 10^{9}$ year) and more chaotic process in which the Milky Way was gradually built through the merging of several small protogalactic "fragments". This is called a bottom-up scenario, in which galaxies are generally formed through the amalgamation of smaller fragments. However, Chiba and Beers (2000) found that the density distribution of the halo changes from highly flattened in the inner parts to nearly spherical in the outer parts with no discrete boundary. Also they found a continuous negative rotational velocity gradient with height above the Galactic plane, both results that are hard to arrange with the original Searle and Zinn (1978) scenario, because chaotic merging would not produce such kinematic and spatial structures.

Therefore, both simplistic models cannot fully explain the distribution, kinematics and abundances of halo stars in the Milky Way. However, the stellar halo only accounts for $\sim 1 \%$ of all stars in the Milky Way, and a more general approach towards understanding the formation of the Milky Way, and disk galaxies in general, is needed. Such an approach has to take into account cosmological principles and the nature and properties of dark matter. Dark matter is the dominant form of matter in the Universe and it should play the key role in galaxy formation through gravitational collapse (Press and Schechter 1974; White and Rees 1978). Numerical and semi-analytic models show that, if the random velocities of the dark matter particles are small compared to the speed of light (cold dark matter or CDM), present day galaxies can be formed through gravitational collapse of the small density fluctuations which where present in the early Universe after the big bang. While the average density of the cosmic matter declined as the Universe expanded, some density enhancements of sufficient size became more pronounced, because of their own 
gravitational attraction. These protosystems drew in more matter from surrounding regions and subsequently merged to larger and larger structures, increasing further the lumpiness of the once highly (but not perfectly) uniform distribution of matter. The "trunks" of such "merger trees" in the hierarchical scenario are the present day galaxies (e.g., Katz 1992; Cole et al. 1994; Mo et al. 1998; Steinmetz and Navarro 1999; Bekki and Chiba 2001; Steinmetz and Navarro 2002). Although these simulations lack high enough resolution to study the detailed spatial and kinematic distribution of stars, they qualitatively can explain the formation and properties of different galactic components. Recent simulations of galaxy formation in the $\Lambda \mathrm{CDM}$ cosmology were able to build Milky Way-like spiral galaxies including thin disk, thick disk, bulge and halo (Abadi et al. 2003a,b; Brook et al. 2004b; Samland 2004; Governato et al. 2007).

The hierarchical merging scenario predicts a richly structured phase-space distribution of dark matter and stars (e.g., Bullock and Johnston 2005). Early evidence for remnants of accretion events in the outer halo was found by, e.g., Doinidis and Beers (1989, five stars at $\langle d\rangle \sim 5 \mathrm{kpc}$ distance from the sun), Arnold and Gilmore (1992, four stars at $d \sim 30 \mathrm{kpc}$ ), Ibata et al. (1994, discovery of the disrupting Sagittarius dwarf galaxy as a clump in radial velocity space of several $100 \mathrm{~K}$ and $\mathrm{M}$ giants) or Majewski et al. (1996, several metallicity/phase space clumps in a sample of 154 stars at $d \lesssim 8 \mathrm{kpc}$ ). Majewski et al. (1996). The last years have seen a dramatic increase in the detection of halo substructure thanks to automatic large-scale photometric surveys such as 2MASS (Skrutskie et al. 2006) and SDSS (Stoughton et al. 2002). Prominent examples are the currently accreted and disrupted Sagittarius dwarf galaxy with its associated tidal tails (Ibata et al. 2001; Majewski et al. 2003; Belokurov et al. 2006a), the Orphan stream (Belokurov et al. 2006a), the Virgo overdensity (Vivas et al. 2001; Newberg et al. 2002; Juric et al. 2008) or the globular clusters Palomar 5 (Odenkirchen et al. 2001; Grillmair and Dionatos 2006) and NGC 5466 (Belokurov et al. 2006b; Grillmair and Johnson 2006).

Numerical simulations predict that most accreted satellites would also spread their tidal debris on eccentric orbits into the Milky Way's inner halo (Helmi and White 1999) or even the disk component (Abadi et al. 2003b; Meza et al. 2005); thus we can expect to find such "fossil remnants" right in our immediate surroundings. Although no longer spatially coherent, such stellar streams keep their common origin imprinted into their chemical and dynamical properties. This allows us to study the formation history of our Galaxy by disentangling its individual "building blocks" from the phasespace distribution of solar neighborhood halo stars, for which we have much better kinematic and abundance informations than for their distant counterparts. The difficulty, however, lies in the very low density contrast that is expected for individual streams. Due to the short dynamical time scales near the disk, a single progenitor can dispose multiple streams in the solar neighborhood in a short period of time, leading to an expected total of several hundreds of cold $\left(\sigma \lesssim 5 \mathrm{~km} \mathrm{~s}^{-1}\right)$ stellarstreams (Helmi and White 1999; Helmi et al. 2003; Gould 2003). ${ }^{1}$ For obtaining most of the

\footnotetext{
1 Compare this number to an expected number of tens of thousands of CDM particle streams (Helmi et al. 2003; Vogelsberger et al. 2008).
} 
informations hidden in the phase-space structure of local stars it follows that (i) a large sample of thousands of halo stars with very accurate velocities would be needed and (ii) the strategies for finding the individual streams and deriving informations about the past should be optimized. The first point is currently addressed by large-scale surveys such as RAVE (Steinmetz et al. 2006) and SEGUE (Yanny et al. 2009) and will be completely satisfied when the Gaia mission (Perryman 2001) is completed. The second point is part of this review, which should be seen both as an introduction for people new to this field as well as a reference summarizing the current state of research.

\section{The formation of stellar halo streams}

In this section I will summarize the main processes that lead to the formation of stellar halo streams that might show up in samples of nearby halo stars.

\subsection{Tides and dynamical friction}

Two effects are important for accreted satellites to reach the inner halo and the disk. The first is dynamical friction. As the satellite moves through the fluid of lighter particles of the host halo, they get pulled towards the satellite, which in the meantime moves along. The resulting overdensity or "gravitational wake" behind the satellite leads to a steady deceleration of the latter. The deceleration of a satellite of (point) mass $M_{\mathrm{S}}$ moving at velocity $\boldsymbol{v}_{s}$ on a straight orbit through an infinite, homogeneous and isotropic field of particles with mass density $\rho_{\text {host }}$ can be expressed through Chandrasekhar's analytic formula (Chandrasekhar 1943):

$$
\frac{\mathrm{d} \boldsymbol{v}_{\mathrm{s}}}{\mathrm{d} t}=-4 \pi G^{2} M_{\mathrm{s}} \ln \Lambda \rho_{\mathrm{host}}\left(<v_{\mathrm{s}}\right) \frac{\boldsymbol{v}_{\mathrm{s}}}{\left|\boldsymbol{v}_{\mathrm{s}}\right|^{3}} .
$$

Here, $G$ is the gravitational constant, $\rho_{\text {host }}\left(<v_{\mathrm{S}}\right)$ the density of field particles at the position of the satellite with velocities less than $\left|\boldsymbol{v}_{\mathrm{s}}\right|$ and $\ln \Lambda$ is the Coulomb logarithm, named so in analogy with the equivalent logarithm in the field of plasma physics. Note that the drag force, $M_{\mathrm{s}} \frac{\mathrm{d} v_{\mathrm{s}}}{\mathrm{d} t}$, is proportional to $M_{\mathrm{S}}^{2}$, so that massive satellites loose their energy and angular momentum much faster than low massive ones. Equation (1) further predicts the merging timescale to depend on the masses of the satellite and host halo as (e.g., Binney and Tremaine 2008, Eq. 8.13)

$$
\tau_{\text {merge }} \propto\left(\ln \Lambda \frac{M_{\mathrm{s}}}{M_{\text {host }}}\right)^{-1} .
$$

Besides assuming linear motion of a point mass through an uniform and isotropic background of particles, Chandrasekhar's formula also neglects any gravitational attraction of these particles for one another. Therefore, in general, Eqs. 1 and 2 can not describe accurately the orbital decay of extended, mass-loosing satellites moving on eccentric orbits within a live host halo in $N$-body simulations. For example, while 
Sales et al. (2007) found that the infall timescales of satellites onto host halos in their $N$-body/gasdynamical simulations follow the trend with mass ratio given by Eq. (2), Boylan-Kolchin et al. (2008) found a non-linear dependence in their $N$-body simulations scaling as $\left(M_{\mathrm{S}} / M_{\text {host }}\right)^{-1.3}$ (i.e., the satellite sinking more slowly than predicted by the standard formula). Another uncertainty is the choice of the Coulomb logarithm. The term $\ln \Lambda$ is given by

$$
\ln \Lambda=\ln \left(\frac{b_{\max }}{b_{\min }}\right),
$$

where $b_{\min }$ and $b_{\max }$ denote the maximum and minimum impact parameters, i.e., the maximum and minimum distances of closest approach between the satellite galaxy and the field stars of the host galaxy. Usually, $b_{\min }$ is set to the size of the satellite galaxy (i.e., its virial radius, White 1976), while the choice of $b_{\max }$ is less clear and somewhat arbitrary (Binney and Tremaine 2008, Sect. 8.1). Hashimoto et al. (2003) argued that $b_{\max }$ should be varied according to the actual separation of the satellite from the host galaxy's center in order to account for the density gradient of the host halo in $N$-body simulations. However, Fujii et al. (2006) showed that this approach predicts too fast sinking times of satellites which suffer significant mass loss, because recently stripped particles would contribute to the drag on the satellite.

Mass loss of the satellite is the second important effect and occurs primarily at each pericentric passage through tidal shocks, but also at other parts of the orbit due to the limitation of bound satellite particles to stay within the tidal surface. If the satellite is on a circular orbit, Jacobi-Hill theory predicts that stars with Jacobi energy $E_{J}=\frac{1}{2} v^{2}+\Phi_{\text {eff }}$ stay bound to the satellite, if they lie inside the last closed zero velocity surface, the so-called Roche surface, at which $E_{J}$ equals the effective potential (Fig. 1; Binney and Tremaine 2008, Sect. 8.3). The latter is given through $\Phi_{\text {eff }}(\boldsymbol{r})=\Phi(\boldsymbol{r})-\frac{1}{2}|\omega \times \boldsymbol{r}|^{2}$, where $\Phi(r)$ is the gravitational potential and the second term describes the centrifugal force with the angular speed $\omega$ of each mass around the common center of mass. The Roche surface includes the two Lagrange points $\mathrm{L}_{1}$ and $\mathrm{L}_{2}$, which are saddle points of the effective potential and are inversion symmetric with respect to the satellite's center only for very small satellite masses (Choi et al. 2007). Stars bound to the satellite and having Jacobi energy $E_{J}$ can escape the satellite, if weak perturbations elevate $E_{J}$ above $\Phi_{\text {eff }}\left(\mathrm{L}_{1}\right)$ or $\Phi_{\text {eff }}\left(\mathrm{L}_{2}\right)$. So, mass loss occurs near the two Lagrange points, leading to the formation of a leading and trailing tidal tail. Such tails can be observed as a spectacular example stretching from the Sagittarius dwarf spheroidal (Ibata et al. 2001; Majewski et al. 2003; Belokurov et al. 2006a) or the globular cluster Palomar 5 (Odenkirchen et al. 2001; Grillmair and Dionatos 2006). The leading tail contains stars at lower energies than the trailing tail, so each time stars get stripped from the satellite, e.g., through tidal shocks at pericenter, roughly two groupings of stars at distinct energies result (Johnston 1998; Meza et al. 2005; Choi et al. 2007). Warnick et al. (2008) proposed that this energy difference could be used for the distinction between leading and trailing stars even after significant dispersion throughout the Galaxy. As pointed out by Choi et al. (2007), (dark matter) particles in the leading tail continue to be decelerated by their satellite's gravity and fall towards the central regions of the host. It has not been studied yet, however, if this 


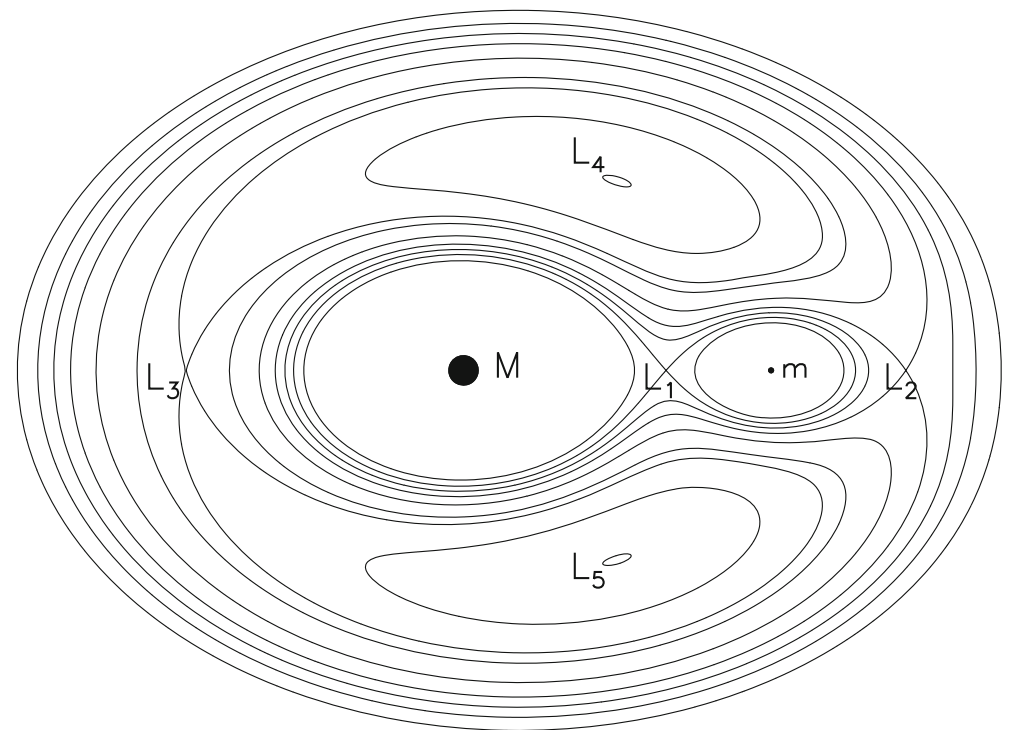

Fig. 1 Contours of constant effective potential in the restricted three-body problem (Jacobi-Hill theory) for satellite-to-host halo mass ratios $m / M=1 / 5$. Note the non-circular form of the Roche surface, which contains $\mathrm{L}_{1}$, and the asymmetry of $\mathrm{L}_{1}$ and $\mathrm{L}_{2}$ with respect to $m$. Stars with $E_{J}<\Phi_{\mathrm{eff}}\left(\mathrm{L}_{1}\right)$ are bound to the satellite

could provide another mechanism for stars to populate the inner halo, even if their satellite stays hung up in the outer halo.

\subsection{The contribution to the inner halo}

Both the adiabatic growth of the host halo and the energy loss of the satellite through dynamical friction have the effect of its orbit spiraling in towards the Galactic center. There is still debate whether the orbits also circularize during infall, and if so, whether dynamical friction causes this circularization (e.g., Colpi et al. 1999; Gill et al. 2004). However, the decay of the satellite's orbit leads to a stronger tidal field and increases the mass loss, which in turn decreases dynamical friction. Using a fully analytical model, Zhao (2004) estimated this interaction between dynamical friction and mass loss to delay the merging of typical present day satellites $\left(10^{7}-10^{9} M_{\odot}\right)$ with initial distances $d>20 \mathrm{kpc}$ to more than a Hubble time. In support of this, Colpi et al. (1999) already argued that the effect of mass loss outperforms dynamical friction and makes it unlikely that remnants of satellites with $M_{\mathrm{s}} / M_{\text {host }}>0.1$ have reached the inner halo yet.

Numerical simulations of the hierarchical buildup of Milky Way-like galaxies in the $\Lambda \mathrm{CDM}$ context seem to support these dynamical friction arguments. A consistent finding is that the inner halo consists of particles stripped from a few significant contributors. These are more massive and have been accreted much earlier ( $\gtrsim 8$ Gyr ago) 
than the satellites that remain today (Bullock and Johnston 2005; Font et al. 2006; Sales et al. 2007; Kazantzidis et al. 2008; Read et al. 2008), although the possibility exists that disruption of the massive satellites is not yet completed and remnants still exist in the inner halo (Cooper et al. 2010). Stated otherwise, "the 'building blocks' of the stellar halo were on average more massive and were accreted and disrupted much earlier than the population of satellites that survive until the present." (Sales et al. 2007). So, in a cosmological context, the presently observed satellites are not assumed to be representative for the ones already accreted into the inner halo. Therefore, it is not surprising that present-day satellites exhibit different chemical compositions than halo stars. The latter tend to have higher $[\alpha / \mathrm{Fe}]$ ratios at a given $[\mathrm{Fe} / \mathrm{H}](\mathrm{Venn}$ et al. 2004), where $\alpha$ stands for the average abundance of the elements $\mathrm{Mg}, \mathrm{Si}, \mathrm{Ca}$ and $\mathrm{Ti}$. This points towards rapid star formation in their progenitor systems, because the $\alpha$ elements are mainly produced in type II supernova ( $\mathrm{SN}$ ) explosions on a short timescale $\left(\sim 10^{7}\right.$ year), while only after a longer timescale of $\sim 10^{9}$ years SNeIa start to lower $[\alpha / \mathrm{Fe}]$ again through expelling additional Fe into the ISM. Recently, however, Nissen and Schuster (2010) reported a dichotomy of nearby halo stars $(\langle d\rangle=115 \mathrm{pc})$ with respect to $[\alpha / \mathrm{Fe}]$, with the high- $\alpha$ stars being more gravitationally bound than the low- $\alpha$ ones. This could indicate that low mass dwarf galaxies have contributed to the inner halo, although to what extend is still to be determined. A possible mechanism for dwarf galaxies to reach the inner halo is infall in massive, loosely bound groups, as pointed out by Li and Helmi (2008). According to Read et al. (2008), this allows even low mass satellites to merge into the inner halo in less than a Hubble time. Read et al. also have shown that — assuming isotropic infall—a typical Milky Way halo will have one out of three mergers at a given mass within $20^{\circ}$ inclination to the disk plane. While the high-inclination satellites would primarily contribute to the Milky Way stellar halo (Bullock and Johnston 2005), the low-inclination ones would be dragged completely into the disk plane by dynamical friction (Fig. 6 in Read et al. 2008), which results in a richly structured accreted thick disk (see also Abadi et al. 2003b; Meza et al. 2005). In all cases the encounters would heat the pre-existing thin disk $^{2}$ and produce several morphological features like bars, flares and warps (Abadi et al. 2003b; Read et al. 2008; Villalobos and Helmi 2009), or even overdensities of stars at distinct rotational velocities that travel as waves through velocity space (Minchev et al. 2009).

It is interesting to ask how one can distinguish between accreted and in situ thick disk stars in a metal-poor local sample. This would give us clues about the fraction of accreted stars versus those that were born within the Milky Way's potential well and contributed to the thick disk through other processes. Mainly three such processes have been discussed in the literature: (i) heating of a pre-existing thin disk (e.g., Villalobos and Helmi 2008; Kazantzidis et al. 2008), (ii) radial mixing, i.e., migration of stars both from inner and outer radii through the solar neighborhood (Sellwood and Binney 2002; Haywood 2008; Schönrich and Binney 2009), or (iii) in situ star formation during a

\footnotetext{
2 Thereby only very massive high-redshift satellites would be able to form a thick disk from a thin disk through heating (Kazantzidis et al. 2008).
} 
gas rich merger (Brook et al. 2004b). By directly comparing the orbital properties of stars from four simulations of these thick disk formation scenarios, Sales et al. (2009) found clear differences in the eccentricity distribution between the generated thick disks. While accreted stars always contribute to the high-eccentricity tail of the distribution, in situ stars have mainly low eccentricities $e \sim 0.2-0.3$, regardless which of the mechanisms (i) to (iii) gave them thick disk properties. Given that a certain fraction of stars in the Solar neighborhood has highly eccentric orbits (Chiba and Beers 2000) and assuming that they have indeed been accreted, we can further ask if we can trace back any of theses stars to a common origin. In other words, do any of these stars belong to the same stellar streams? Assuming a halo solely built through accretion, Helmi and White (1999) predicted the number of individual streams passing the solar neighborhood to be

$$
N_{\text {streams }} \sim[300-500](t / 10 \mathrm{Gyr})^{3},
$$

where $t$ is the time since disruption. In reference to the dynamical friction arguments made above, these streams are expected to stem mainly from a few progenitors only. The Milky Way seems to have a quiet merger history since $t \sim 10-12$ Gyr (corresponding to the age of the thick disk). In such a time any debris from an accreted satellite is expected to be spread over several tens of kpc. Therefore, one cannot expect to find many of the halo streams predicted by Eq. (4) as spatially coherent structures in the solar neighborhood (Seabroke et al. 2008), although hints for a spatial clumping of one kinematically identified substructure have been found (Klement et al. 2009). Instead, other search strategies for stellar streams have to be applied, which I describe in the following section.

\section{Search strategies for halo streams}

\subsection{Integrals of motion and action-angle variables}

The collisionless Boltzmann equation

$$
\frac{\mathrm{d}}{\mathrm{d} t} f(\boldsymbol{r}, \boldsymbol{v}, t)=\frac{\partial f}{\partial t}+\sum_{i=1}^{3}\left(v_{i} \frac{\partial f}{\partial x_{i}}-\frac{\partial \Phi}{\partial x_{i}} \frac{\partial f}{\partial v_{i}}\right)=0
$$

is the fundamental equation of stellar dynamics. It assumes that encounters between stars can be ignored so that every star moves under a mean gravitational potential $\Phi(\boldsymbol{r}, t)$ (therefore the term "collisionless"), where $\boldsymbol{r}=\left(x_{1}, x_{2}, x_{3}\right)$, and $\boldsymbol{v}=\left(v_{1}, v_{2}, v_{3}\right)$ denote the position and velocity vector of a star with respect to the center. $f(\boldsymbol{r}, \boldsymbol{v}, t)$ is the distribution function describing the density of stars in phase-space similar to the distribution of atoms in a gas. Equation (5) states that the phase-space density $f$ around the phase-space point of any particular star is constant. It follows that, as a sample of stars which is initially concentrated into a small phase-space volume (like in a satellite galaxy) disperses in configuration space, at any particular point in phase-space they will grow together kinematically in an infinitesimal volume around 
that point. For example, Helmi and White (1999) have shown that the velocity dispersions at each point along the track of a single stream of stars lost at the same passage decrease overall with $t^{-1}$ with some periodic oscillations due to the spatial density enhancements at the turning points of the orbit. An observer, however, will always measure the coarse-grained phase-space density $\langle f\rangle$, which is the average phase-space density in his finite observational volume. In the inner halo, where orbital timescales are short, $\langle f\rangle$ includes the contribution from multiple streams of a single progenitor. In a given local volume, we eventually will find stars belonging to each of these streams, while an infinitesimal volume only contains stars belonging to one individual stream. This is known as phase mixing. It is caused by the fact that for any individual stream, the phase-space regions with initially high density stretch out and get thinner; the coarse-grained density decreases with time until a final equilibrium state is achieved (e.g., Fig. 1 in Tremaine 1999). Phase mixing means in practice that in a given volume the stellar density of tidal debris from a satellite decreases and its velocity dispersion increases with time, so that individual streams can no longer be separated in velocity space. The broadening in the velocity distribution is caused by the initial differences in the orbital phases between stars stripped at different epochs. In the disk for example the broadening in the velocity distribution is most prominent in the vertical $W$-component, because the vertical frequency with respect to the disk is shorter than the horizontal frequencies. For the angular $V$-component, Helmi and White (1999) calculated that complete phase mixing would give rise to an observed velocity dispersion of $50-150 \mathrm{~km} \mathrm{~s}^{-1}$, depending in proportion on the initial velocity dispersion of the satellite.

The problem of phase mixing can be avoided, if one switches to integrals-of-motionspace (IoM-space). An integral of motion $I(\boldsymbol{r}, \boldsymbol{v})$ is a function of the phase-space coordinates that is constant along any stellar trajectory in phase-space. It does not depend on time:

$$
I(\boldsymbol{r}(t), \boldsymbol{v}(t))=\text { const. }
$$

An integral of motion is called isolating if it isolates the points on a star's trajectory from neighboring points in phase space, contrary to non-isolating integrals which are infinitely many-valued (for more details see e.g., Ollongren 1962; Binney and Tremaine 2008). Classical isolating integrals of motion include the energy $E$ in every static potential, the three components of the total angular momentum in a spherically symmetric potential, or one of its components, e.g., $L_{z}$, in an axisymmetric potential. According to Jeans theorem, in a steady state any function of $n$ integrals of motion automatically solves the collisionless Boltzmann equation (5), because

$$
\frac{\mathrm{d}}{\mathrm{d} t} f\left(I_{1}(\boldsymbol{r}, \boldsymbol{v}), \ldots, I_{n}(\boldsymbol{r}, \boldsymbol{v})\right)=\sum_{i=1}^{n} \frac{\partial f}{\partial I_{i}} \frac{\mathrm{d} I_{i}}{\mathrm{~d} t}=0
$$

The coarse-grained phase-space density-written as a function of the integrals of motion-evolves asymptotically to a state that satisfies Jean's theorem. Therefore, a 


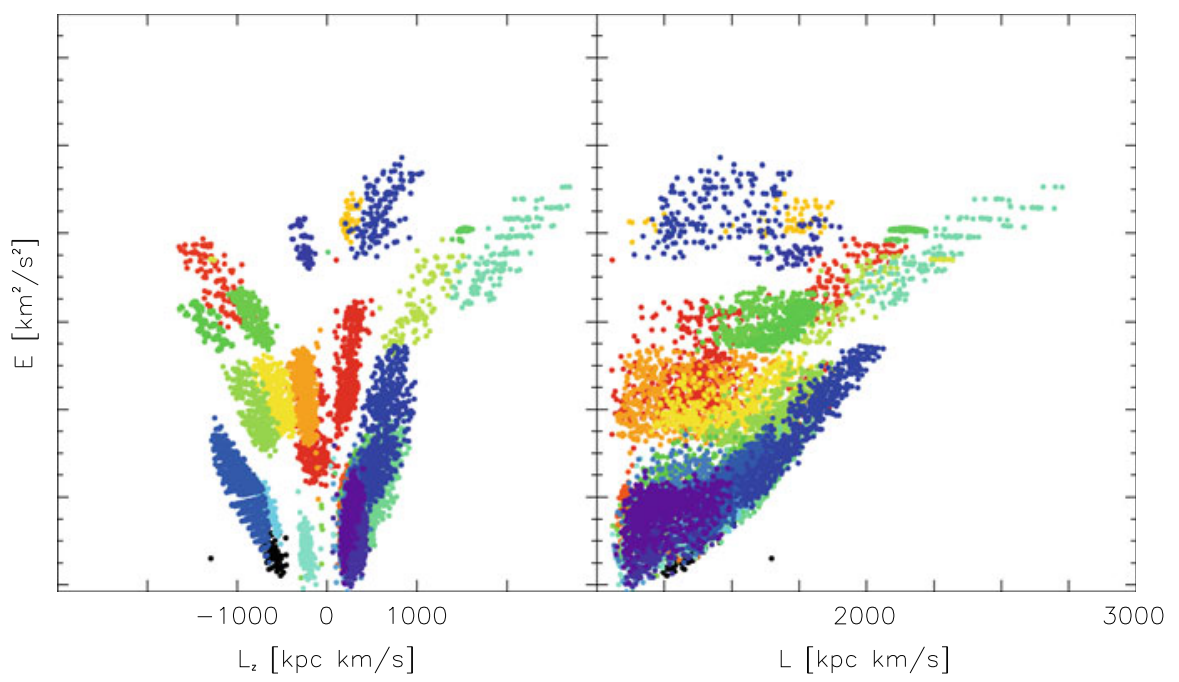

Fig. 2 Simulated $\left(E,|\boldsymbol{L}|, L_{z}\right)$-distribution of stars that have been stripped from 33 different (color-coded) satellites and observed 12 Gyr later within $2 \mathrm{kpc}$ from the sun. Simulated errors as expected for the Gaia mission have been added to the data. Same data as in (Helmi and de Zeeuw, 2000, Fig. 4), except for the smaller size of the observational volume

subpopulation of stars initially confined to a small phase-space volume will remain clumped in the IoM-space (Fig. 2).

Additional insights can be gained from studying the dispersion of stream particles in action-angle coordinates $(\boldsymbol{J}, \boldsymbol{\Theta})$ (Tremaine 1999; Helmi and White 1999; Binney and Tremaine 2008). In Hamiltonian mechanics, actions and angles form a special set of canonical variables that lead to a simple form of the canonical equations of motion:

$$
\frac{\mathrm{d} \Theta}{\mathrm{d} t}=\frac{\partial H(\boldsymbol{J})}{\partial \boldsymbol{J}} \equiv \boldsymbol{\Omega}(\boldsymbol{J}) \quad \frac{\mathrm{d} \boldsymbol{J}}{\mathrm{d} t}=-\frac{\partial H(\boldsymbol{J})}{\partial \boldsymbol{\Theta}}=0,
$$

where the components of $\boldsymbol{\Omega}(\boldsymbol{J})$ are the orbital frequencies. Neglecting the influence of the satellite's potential on the individual stars, the actions $\boldsymbol{J}$ of a star remain constant, while the angles increase linearly with time. The cloud of escaped stream particles, therefore, spreads in only three of the six phase space dimensions at rates which can be shown to depend on the eigenvalues of the Hessian matrix of the Hamiltonian ${ }^{3}$ and the initial spread in the actions. For Stäckel potentials that resemble disk-like flattened axisymmetric potentials, it can be shown analytically that the actions are functions of the three isolating integrals $E, L_{z}$ and $I_{3}$. Again it follows that the stream stars remain clumped in the space of these integrals, even if the potential underwent slow changes in the past. The reason is that usually two or three action variables are adiabatically invariant (like $J_{\Phi}=L_{z}$ in an axisymmetric potential).

Attempts to use the action-angle variables directly to search for fossil remnants of past accretion events have until now only been made sparsely (e.g. by Chiba and

3 The elements of the Hessian matrix are given by $H_{i j} \equiv \frac{\partial^{2} H}{\partial J_{i} \partial J_{j}}$. 
Fig. $3 \Omega$-space distribution of stream particles lost from a satellite without modeled self-gravity on an orbit in a spherical static potential. Grey dots show the distribution of all satellite particles after $10 \mathrm{Gyr}$, whereas the black dots only show the particles inside a sphere of $4 \mathrm{kpc}$ radius. Note the alignment of the patterns along lines of constant frequencies $\Omega_{r}$ and $\Omega_{\Phi}$. Adopted from (Gómez and Helmi, 2010a, Fig. 5)

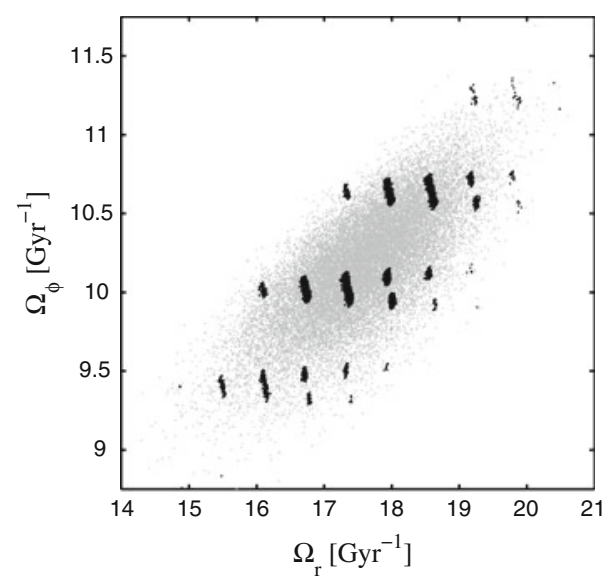

Beers, 2000). The reason might be that action-angle variables are calculated readily only for integrable, time-independent potentials, while for real stars in the unknown Galactic potential they have to be constructed via sophisticated numerical methods (e.g., Binney and Spergel 1984; McGill and Binney 1990). Theoretically, the space of orbital frequencies seems to be a promising one (McMillan and Binney 2008; Gómez and Helmi 2010a). In a static potential, stars stripped at time $t=0$ and observed at time $t$ can be identified as distinct clumps-each representing an individual stream and separated by regular distances $\delta \Omega_{i}$ that directly measure $t$ through $\delta \Omega_{i}=\frac{2 \pi}{t}$ (Fig. 3). The reason is that any observational volume, such as the solar neighborhood, only picks out those stars that have moved a certain amount in an angular direction, e.g., azimuth $\Phi$, plus or minus an integer number of completed angular periods, like rotations about the Galactic center. The number of these integers is given by $\Omega_{i} t / 2 \pi$, so the number of patches in $\boldsymbol{\Omega}$-space ${ }^{4}$ increases proportional with $t^{2}$. Meanwhile, they get smaller proportional to $t^{-2}$, because the width in one dimension is given by $\Delta \Theta_{i} / t$, where $\Delta \Theta_{i}$ is the range in angle component $i$ which in turn is determined by the size of the observational volume (McMillan and Binney 2008). Gómez and Helmi (2010a) have shown that also for a time-evolving host potential and even a N-body simulation of the accretion of a satellite onto a live disk, the clumps in frequency space are conserved, although their regularity breaks down in the latter case. In addition, the timescale of growth of the host potential seems to leave its imprint as a curvature in the alignment of the patches - a clear advantage over the classical integrals $E$ and $L_{z}$, that according to Peñarrubia et al. (2006) can only constrain the present Galactic potential. Recently, Gómez and Helmi (2010b) applied the stream search in frequency space to a mock Gaia catalog that contained tidal debris from 42 disrupted satellites. In their simulations, these authors included observational errors expected for Gaia as well as a time-varying potential of the halo, bulge and disk. Gómez and Helmi found that only

\footnotetext{
${ }^{4}$ Usually, the vertical frequency $\Omega_{z}$ is omitted because $\Omega_{z} \gg \Omega_{R} \wedge \Omega_{z} \gg \Omega_{\Phi}$, implying that the number of streams in the $z$-direction is much larger, and so after some time, much harder to see unless one has very high numerical resolution (or the orbit is elongated in the $z$-direction).
} 
for small observational errors (relative parallax errors less than 0.02), a discrimination between adjacent streams in frequency space and a clear determination of the time since accretion are possible. In their simulations, they were able to compute accretion times for $\sim 30 \%$ of the identified satellites, accurate to $15-25 \%$. The estimated times are always lower limits and the difference to the actual time since accretion depends on the form of the gravitational potential and its evolution.

The actions $\boldsymbol{J}$, besides having the advantage of being constant and adiabatically invariant once the influence of the satellite's gravitation has ceased, also allow the identification of individual streams as different patches (McMillan and Binney 2008). However, the patches are not aligned along lines of constant $J_{i}$ like in the case of the $\Omega_{i}$ s (in a static potential), because the $\Omega_{i}$ s depend on all three $J_{i}$ s, so that the constraints on $\boldsymbol{\Omega}$ also affect $J_{z}$. Therefore, Gómez and Helmi (2010a) found that individual streams are less clearly separable in $\left(J_{r}, J_{\Phi}\right)$-space, comparable to the space of classical integrals $\left(E, L_{z}\right)$. However, the large expected number of streams passing the solar neighborhood given by Eq. (4) could make it difficult to detect individual streams from a given satellite, especially when observational errors are considered. Therefore, in practice, it might be better to use the classical integrals of motion $\left(E,|\boldsymbol{L}|, L_{z}\right)$ to first detect tidal debris, because individual streams of a satellite roughly contribute to the same clump in IoM-space, raising the chances of identifying the individual satellites. Afterwards, $\boldsymbol{\Omega}$-space could be used to compute the time since accretion (Gómez and Helmi 2010b).

Indeed, the classical integrals of motion have been used successfully for finding tidal debris in the solar neighborhood (Helmi et al. 1999; Chiba and Beers 2000; Re Fiorentin et al. 2005; Kepley et al. 2007; Smith et al. 2009); however, some limitations have to be considered (pointed out by Helmi et al. 2006): First, the total angular momentum $|\boldsymbol{L}|$ is only strictly conserved in a spherical potential which is not the case for the Milky Way. However, this seems to be a minor point, because even for stars that move in axisymmetric flattened potentials, $|\boldsymbol{L}|$ is approximately conserved and similar to the third integral $I_{3}$, for which no analytic expression exists (Binney and Tremaine 2008, Sect. 3.2.2). Also, Chiba and Beers (2000) showed that the distribution of halo stars in the space spanned by isolating integrals of motion in an aspherical Stäckeltype potential can be closely mapped into the IoM-space of a spherical potential. A second concern is that continuing accretion and merging events would have changed the potential steadily and altered the energy of an orbiting satellite. This emphazises the advantages of adiabatic invariants such as the actions (among them $J_{\Phi}=L_{z}$ ). However, the clumpiness of the local IoM-space (Morrison et al. 2009; Klement et al. 2009) suggests that (i) violent relaxation might have had a less smoothing effect on the signatures of the inner halo stars than expected and/or (ii) only a couple of early major mergers have contributed to the inner halo, in agreement with predictions from $\Lambda C D M$ simulations and a direct effect of dynamical friction acting primarily on the most massive satellites (e.g., Helmi et al. 2003, and Sect. 2.2 in this review). Both Dynamical friction and the gravitational influence of the satellite also lead to the problem that stars lost at different passages end up having different energy levels, in this way creating multiple signatures in the $\left(E, L_{z}\right)$-space (Meza et al. 2005; Choi et al. 2007). Finally, to calculate the energy, one needs the full 6D phase space 
information $(\boldsymbol{r}, \boldsymbol{v})$, which can be observed, plus the potential $\Phi(\boldsymbol{r})$, which is desired, but not directly observable.

Therefore, alternative "effective" integrals of motion have been proposed (Helmi et al. 2006; Arifyanto and Fuchs 2006; Dettbarn et al. 2007; Klement et al. 2008, 2009). The idea behind such approaches is to find parameters that characterize stellar orbits and can be easily expressed through the observables. For example, Helmi et al. (2006) proposed to look for stellar streams in a space spanned by the apocenter, pericenter and angular momentum $L_{z}$ (the APL-space). Stellar streams then cluster around lines of constant eccentricity. However, to calculate the eccentricity, a guess of the true potential is required. Arifyanto and Fuchs (2006), Dettbarn et al. (2007), Klement et al. (2008) and Klement et al. (2009) followed a similar approach in the sense that they tried to find stars with the same orbital eccentricity. Their strategy in finding nearby stellar streams in velocity space is based on the Keplerian approximation for orbits developed by Dekker (1976); in this approximation, the potential $\Phi(R)$ is expanded with respect to $\frac{1}{R}$, and the effective potential, $\Phi_{\text {eff }}(|\boldsymbol{L}|, R) \equiv \Phi(R)+|\boldsymbol{L}|^{2} /\left(2 R^{2}\right)$, takes on a form similar to the one in the Kepler problem. The assumption is that stars in the same stellar stream move on orbits that stay close together, which is justified by numerical simulations of satellite disruption (Helmi et al. 2006). Further assuming a spherical potential and a flat rotation curve, these stars should form a clump in the projection of velocity space spanned by

$$
\begin{aligned}
v & \equiv \arctan \left(\frac{V+V_{\mathrm{LSR}}}{W}\right), \\
V_{\mathrm{az}} & \equiv \begin{cases}\sqrt{\left(V+V_{\mathrm{LSR}}\right)^{2}+W^{2}} & v \leq 180^{\circ} \\
-\sqrt{\left(V+V_{\mathrm{LSR}}\right)^{2}+W^{2}} & v>180^{\circ},\end{cases} \\
V_{\Delta \mathrm{E}} & \equiv \begin{cases}\sqrt{U^{2}+2\left(V_{\mathrm{LSR}}-V_{\mathrm{az}}\right)^{2}} & v \leq 180^{\circ} \\
\sqrt{U^{2}+2\left(V_{\mathrm{LSR}}+V_{\mathrm{az}}\right)^{2}} & v \leq 180^{\circ} .\end{cases}
\end{aligned}
$$

$v$ is the angle between the orbital plane (which is fixed in a spherical potential) and the direction towards the North Galactic Pole and ranges from $0^{\circ}$ to $180^{\circ}$. Stars with $v>180^{\circ}$ are treated as stars moving on retrograde orbits in a plane with inclination angle $v-180^{\circ} . V_{\mathrm{az}}$ is related to the angular momentum $|\boldsymbol{L}|$ and defines the guiding center radii of the stars, $R_{0}=R_{\odot} \frac{V_{\mathrm{az}}}{V_{\mathrm{LSR}}}$, in the plane labeled by $\nu . V_{\Delta \mathrm{E}}$ is a measure of a star's eccentricity $e$ through

$$
e=\frac{1}{\sqrt{2} V_{\mathrm{LSR}}} V_{\Delta \mathrm{E}}
$$

(Eq. 11 in Klement et al. 2009). Also, it can be shown that $V_{\Delta \mathrm{E}}^{2}$ is adiabatically invariant, because it approximately relates to the radial action integral $J_{R}$ (Eq. 13 in Klement et al. 2009). ${ }^{5}$ Equations (9-11) fully define an orbit in the Keplerian approximation, because its turning points are given by

\footnotetext{
5 In the formalism of Arifyanto and Fuchs (2006) and Klement et al. (2008), slightly different notations have been used, which follow easily under the assumption of motions in the orbital plane $z=0$ or,
} 


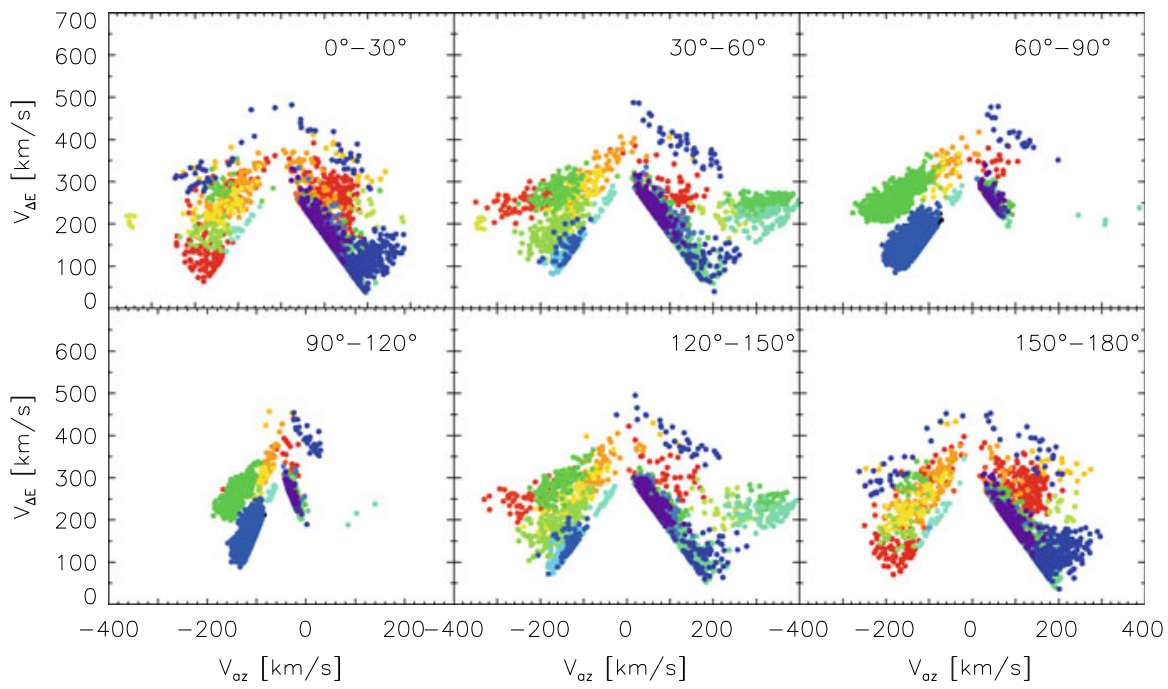

Fig. $4\left(v, V_{\mathrm{az}}, V_{\Delta \mathrm{E}}\right)$-distribution of the same stars as in Fig. 2. The same color coding has been used. The stars have been binned into $30^{\circ}$ wide $\nu$-slices, as indicated in each panel, and displayed in $\left(V_{\mathrm{az}}, V_{\Delta \mathrm{E}}\right)$. Note that these quantities are derived in a spherical potential, although the true potential in this case includes a disk (Helmi and de Zeeuw 2000, Model I). Also note the symmetry of multiple streams from the same satellites with respect to $v=90^{\circ}$, which follows from the symmetry in the $(V, W)$-distribution of phase-mixed tidal debris (Fig. 5 below) and Eq. (9)

$$
\frac{R_{\min }}{R_{0}}=\frac{1}{1+e} \quad \text { and } \quad \frac{R_{\max }}{R_{0}}=\frac{1}{1-e} .
$$

Dekker (1976) has shown that her approximation holds for eccentricities up to $e=0.5$ $\left(V_{\Delta \mathrm{E}}^{2} \approx 150 \mathrm{~km} \mathrm{~s}^{-1}\right)$, although in practice, the clumping in the space spanned by the quantities (9-11) seems to remain even for stronger deviations from circularity (Fig. 4).

\subsection{The use of velocities and positions}

As noted above, stream stars passing the solar neighborhood have very similar azimuthal frequencies or, equivalently, angular momenta. Therefore, these stars show a narrow distribution of azimuthal velocities. In this respect they behave exactly like the classical moving groups in the solar neighborhood, that emerge either from dissolved open clusters or through dynamical resonances with the non-axisymmetric potential. However, moving halo groups often have a distinct banana-shaped $(U, V)$ velocity distribution, which is a consequence of their eccentric orbits (Helmi and White 1999; Helmi et al. 2006; Villalobos and Helmi 2009): The slightly different orbital phases of the stars in the observed volume can result in some stars being at their apocenters

Footnote 5 continued

equivalently, $v=90^{\circ}$ only. Note also that in the studies of Dettbarn et al. (2007) and Klement et al. (2009) only the upper definition of Eq. (11) has been used, which still allows identification of substructure, but not the calculation of eccentricities from Eq. (12). 

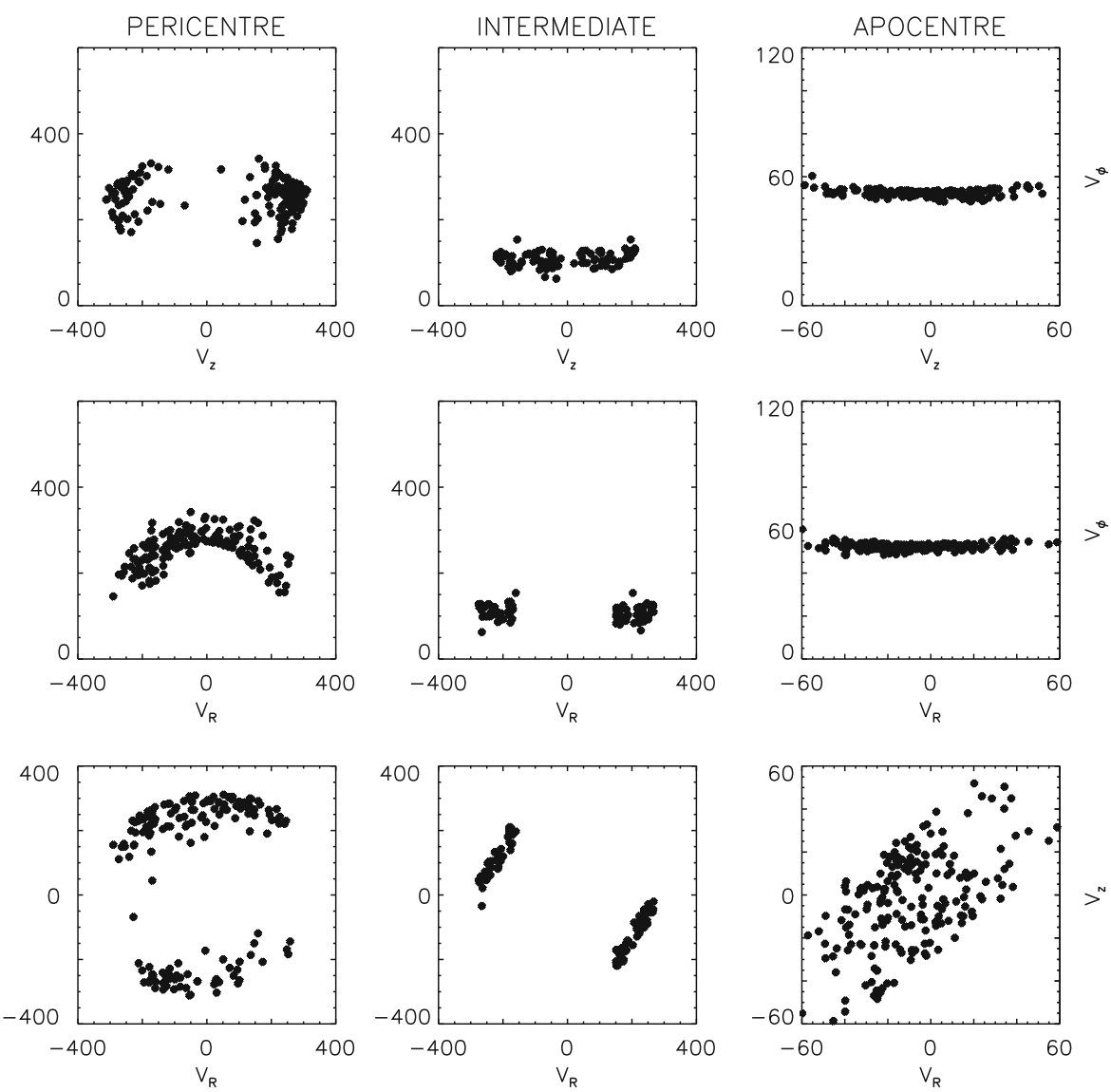

Fig. 5 Simulated velocity space distribution of satellite stars $13.5 \mathrm{Gyr}$ after becoming unbound to their progenitor system. The figure only plots stars within a $3 \mathrm{kpc}$ wide box centered on the progenitor's pericenter $(r=6.0 \mathrm{kpc}$, left panel), apocenter $(r=37.0 \mathrm{kpc}$, right panel) or within (middle panel). Note the banana shape of stars near their apo- and pericenters. Note that in the solar neighborhood $v_{R} \approx U, v_{\phi} \approx V$ and $v_{z}=W$. Adopted from (Helmi and White, 1999, Fig. 5)

$(U=0)$, while the others either move away from $(U>0)$ or towards $(U<0)$ it (Fig. 5). For the same reasons, if the pericenter of some stars lies in the observational volume, a similar, but switched and stronger curved banana pattern results (because stars move fastest near their pericenters). Compared to in situ thick disk stars, accreted stars mostly occupy the outskirts of the $(U, W)$-distribution (Villalobos and Helmi 2009).

An even clearer separation between tidal debris and metal-poor disk stars can be obtained by observing stars out to large cylindrical radii $R$. Assuming a constant rotation curve implies $L_{z} \propto R$ for disk stars, whereas stream stars display a nearly constant angular momentum that depends mainly on the initial conditions of their progenitor's orbit (like the orbital inclination), but not on $R$. Therefore, by plotting $R$ vs. $L_{z}$, the disk stars discriminate themselves from the stream stars by their trend of rising $L_{z}$ as $R$ increases (Fig. 6 inVillalobos and Helmi 2009). 
Fig. 6 Histogram of rotational velocities $V_{\text {rot }}$ for all stars from the Beers et al. (2000) catalog of metal-poor stars (dash-dotted histogram). The distribution is decomposed into three Gaussian components representing the thin disk, thick disk and halo as labeled. Note the excess of stars lagging the LSR with

$\sim 120 \mathrm{~km} \mathrm{~s}^{-1}$ (Arcturus stream) and $\sim 270 \mathrm{~km} \mathrm{~s}^{-1}$

( $\omega$ Cen stream)

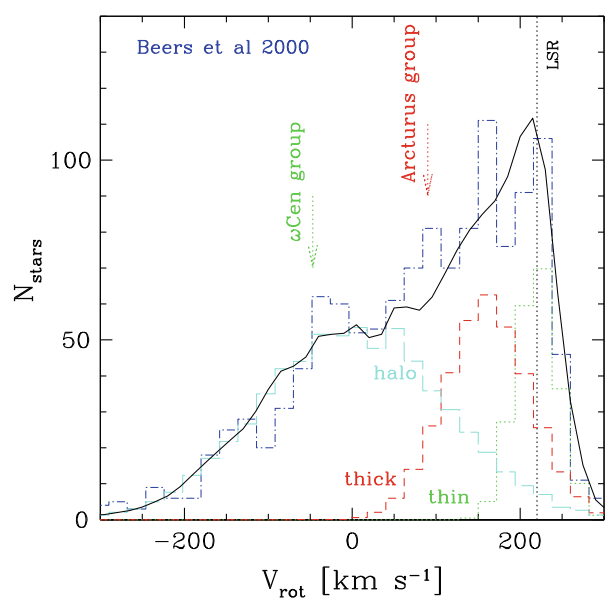

Finally, heliocentric line-of-sight velocities $v_{\text {los }}$, which are much easier to obtain than full space motions, can be used to detect stellar streams against the disk star background. In this case, circular rotation of the disk stars implies a sinusoidal dependence of $v_{\text {los }}$ on galactic longitude $l,{ }^{6}$ while again the stream stars dominate the wings of the $v_{\text {los }}$ distribution and should become apparent after subtraction of the mean disk rotation (Villalobos and Helmi 2009).

\section{Moving halo groups}

\subsection{Current state of research}

\subsubsection{The early years}

Since Proctor (1869) it has been known that among the nearby stars some groups exist that share the same streaming motion, therefore called stellar streams or moving/kinematic groups. It was hypothezised early that such moving groups result from the breakup or dispersion of open clusters (see e.g., Eggen 1958 and references therein), an effect that also would apply to halo globular clusters (Oort 1965), satellite galaxies or other accreted building blocks of the Galaxy like the Searle and Zinn fragments. In 1959, Eggen and Sandage discovered a group of five subdwarfs within 20 pc-among them the star RR Lyrae-with large space motions moving towards a common convergence point. The group was named the Groombridge 1830 group after the star Groombridge 1830 (HR 4550), which lags the Local Standard of Rest with $V \approx-130 \mathrm{~km} \mathrm{~s}^{-1}$. In the years that followed, Eggen extended his observations of subdwarfs in number and distance and established the existence of high-velocity, metal-poor moving groups (e.g., Eggen 1977 and references therein). Two of these, the

\footnotetext{
${ }^{6} v_{\text {los }}=\left(U-U_{\odot}\right) \cos b \cos l+\left(V-V_{\odot}\right) \cos b \sin l+\left(W-W_{\odot}\right) \sin b$; for circular low latitude orbits this implies $v_{\text {los }} \sim\left(V-V_{\odot}\right) \cos b \sin l$.
} 
groups named after Kapteyn's star and Arcturus, have only recently been investigated in great detail (see Sect. 4.1.3). In the early 90s, despite some false detections of halo substructure, the evidence for nearby kinematic halo streams interpreted as tidal debris increased (for a review see Majewski 1993 Sect. 5.1). Tremaine (1993) computed the fractional volume containing one or more tidal streams to be $\approx 1$ at the Solar radius, if the halo would have been built solely through merging and accretion. Ten years later, Gould (2003) should derive from statistical arguments on a set of 4588 halo stars that there are about $\sim 400$ cold streams present in the solar neighborhood, whereby no stream could contain more than $5 \%$ of the local stars. This number is fully consistent with the predictions of Eq. (4) and has until now not been proved false. More possible streams were found when Poveda et al. (1992) analyzed the clustering of 206 halo stars in $\left(E, L_{z},[\mathrm{Fe} / \mathrm{H}]\right)$-space. These authors justified their approach with the argument that "...because of the high incidence of chaotic orbits among halo stars...the usual criterium of identifying group members by the similarity of their $V$-velocities breaks down."

\subsubsection{The Helmi stream}

In 1999, (Helmi et al., 1999, hereafter H99) published their seminal paper on the discovery of two single streams stemming from the same progenitor, that "...had a highly inclined orbit about the Milky Way at a maximum distance of $\sim 16 \mathrm{kpc}$, and it probably resembled the Fornax and Sagittarius dwarf spheroidal galaxies." The two streams are clearly separated in the $(U, V)$-plot, but occupy the same region in the $\left(L_{z}, L_{\perp}\right)$-plane $\left(\sim(1200,2200) \mathrm{kpc} \mathrm{km} \mathrm{s}^{-1}\right.$.) The stream stars are on prograde eccentric orbits $\left(R_{\text {peri }} \sim 7 \mathrm{kpc}, R_{\text {apo }} \sim 16 \mathrm{kpc}\right)$ that reach distances up to $|z|_{\max } \sim 13 \mathrm{kpc}$ above or below the plane. Originally, H99 concluded that about $10 \%$ of the local halo stars would belong to this stellar stream. However, this number was questioned when Chiba and Beers (2000) analyzed 728 stars with full space motions (accurate to $\sim 20 \%$ ) and $[\mathrm{Fe} / \mathrm{H}]<-1$ which were drawn from the Beers et al. (2000) sample of 2106 metal-poor stars without kinematic bias. Although this meant a sample size three times as large as H99's, they found practically the same number of stream members. The Beers et al. catalog was re-examined by Re Fiorentin et al. (2005), but now concentrating on the classical search strategy for moving groups, namely picking out stars with strong correlations in velocity space. Among the 5\% of the fastest moving halo stars, Re Fiorentin et al. found a kinematic group consisting of 3 stars with similar angular momenta, but somewhat lower binding energy than the H99 streams. They hypothezised that their moving group could consist of stars from a same progenitor of the $\mathrm{H} 99$ group, but stripped at an earlier time, before dynamical friction dragged in the satellite more closely. In total, based on their $\left(L_{z}, L_{\perp}\right)$-distributions, Re Fiorentin et al. counted 16 stars out of 410 (3.9\%) as putative members of the H99 streams, consistent with Gould's 2003 estimate that no single stream could contain more than $5 \%$ of the local halo stars. ${ }^{7}$ In this respect, it seems that the H99 progenitor

\footnotetext{
7 Even more so, since it could be expected that the 16 stars from Re Fiorentin et al. are distributed between three $\mathrm{H} 99$ streams, i.e., the kinematic group consisting of 3 stars plus the original two streams with positive and negative $W$ velocities, respectively.
} 
is one of the major contributors to the local halo- a result that has further been supported when Kepley et al. (2007) analysed a sample of 231 nearby ( $d \leq 2.5 \mathrm{kpc}$ ) metal-poor red giants, RR Lyrae and RHB stars, including again a subset of the Beers et al. (2000) catalog. These authors identified 11 or possibly 12 stars connected to the H99 group ( 5\%) and showed that their progenitor was likely accreted between 6 and 9 Gyr ago by comparing the observed asymmetric $W$-distribution of the stars (8/11 with negative $W$ ) to simulations. In addition, $[\alpha / \mathrm{Fe}]$ ratios for three of the H99 stars revealed that they are very similar to other solar neighborhood halo stars, implying formation in a massive progenitor where enrichment occurred mainly through SNe II.

The chemical similarity of the stream stars among each other, but also with normal inner halo stars, was recently shown by Roederer et al. (2010) through determinations of abundances or upper limits, respectively, for 46 elements in 12 kinematically selected member stars from the lists of Chiba and Beers (2000), Re Fiorentin et al. (2005) and Kepley et al. (2007). Roederer et al. found that the stream stars span a broad range in $[\mathrm{Fe} / \mathrm{H}]$ from -3.4 to -1.5 , but compared to typical present-day $\mathrm{dSph}$ and ultra-faint dwarf galaxies, only small star-to-star dispersions of [X/Fe] at a given metallicity have been found. The high $\alpha$ abundances (e.g., $[\mathrm{Ca} / \mathrm{Fe}]=+0.4)$ together with the trend of the $n$-capture abundances (e.g., $[\mathrm{Ba} / \mathrm{Fe}]$ and $[\mathrm{Eu} / \mathrm{Fe}])$ to increase with metallicity point towards a scenario in which star formation in the H99 progenitor was truncated before SNeIa or AGB stars enriched the ISM.

Four members of the H99 stream with negative $W$ velocities also showed up when Dettbarn et al. (2007) re-analyzed the Beers et al. (2000) data using Dekker's 1976 theory of Galactic orbits and searching for streams in the space spanned by $\left(v, V_{\mathrm{az}}, V_{\Delta \mathrm{E}}\right)$ (see Sect. 3.1). The same search strategy was used by Klement et al. (2009) on a sample of 22,321 nearby $(d \leq 2 \mathrm{kpc})$ subdwarfs with $[\mathrm{Fe} / \mathrm{H}]<-0.5$ from the seventh SDSS data release with astrophysical parameters from SEGUE (Yanny et al. 2009). This sample, being fainter than the Beers et al. compilation, added another 21 stars to the H99 stream. Referred to the total number of likely halo stars in the SDSS sample ( 4400), this only accounts for $0.5 \%$ of the local halo. I should note that the search strategy only picked out these 21 stars from a single stream with negative $W$-velocities, similar to Dettbarn et al.'s study from 2007. If one assumes the asymmetry in the $W$-distribution in the sample of Kepley et al. (2007) as representative, another $3 / 11 \cdot 21 \approx 6 \mathrm{H} 99$ stars with positive $W$ velocities could be expected, presumably too few to appear in the significance maps of Klement et al. (2009). Kepley et al. (2007) also found that $67 \%$ of their H99 member stars lay within $d=1 \mathrm{kpc}$, a range which is incompletely sampled in Klement et al. (2009, Fig. 5c). On the other hand, Smith et al. (2009) predict up to $\sim 7 \%$ of all halo stars within $2.5 \mathrm{kpc}$ to belong to the H99 streams. Their result is based on the fraction of 12 putative H99 members out of 645 SDSS subdwarfs selected from a reduced proper motion diagram, again using the $\left(L_{z}, L_{\perp}\right)$ criterion and correcting for any selection effects due to the reduced proper motion bias. An explanation for these different results could be that the method of Dettbarn et al. (2007) and Klement et al. (2009) identifies single streams from the same progenitor in different regions of $\left(v, V_{\mathrm{az}}, V_{\Delta \mathrm{E}}\right)$-space, requiring a large number of stars in any single stream for a significant detection; in 
contrast, these single streams all enhance the signal in the same region of the $\left(L_{z}, L_{\perp}\right)$ plane.

\subsection{3 $\omega$ Centauri, the Kapteyn and Arcturus streams}

The Beers et al. (2000) catalog, besides containing the H99 stream as the most significant feature, reveals further substructure when plotting a histogram of the rotational velocities of the stars like in Fig. 6. The peak of stars with rotational velocities of $V_{\text {rot }} \sim 100 \mathrm{~km} \mathrm{~s}^{-1}$ was investigated in detail by Navarro et al. (2004) and attributed to the Arcturus stream, first described in 1971 by Eggen (1971) as a moving group of stars with similar kinematics as the star Arcturus. Originally, Navarro et al. (2004) suggested a tidal origin for the Arcturus moving group, supported by the well-defined sequence of abundance ratios of member stars taken from the then available Gratton et al. (2003) catalog. Arifyanto and Fuchs (2006), who later detected the Arcturus group in a compilation of various catalogs using their $\left(V_{\mathrm{az}}, V_{\Delta \mathrm{E}}\right)$ method, suggested an external origin, too, based on the goodness of theoretical 12 Gyr isochrone fits to the putative member stars. The same suggestion was made by Helmi et al. (2006) for a group of stars (their Group 2) detected in the APL-space distribution of GenevaCopenhagen survey stars (Nordström et al. 2004) and possibly related to the Arcturus stream (according to its $V$ - and $[\mathrm{Fe} / \mathrm{H}]$-distribution).

However, recent detailed abundance studies including various $\alpha$ and other elements throw doubt on the hypothesis that the Arcturus stream is composed of a homogeneous stellar population (Williams et al. 2009). Instead, the putative members ${ }^{8}$ do not differ from the surrounding disk stars. This either indicates a progenitor system that had to be very massive to self-enrich to $[\mathrm{Fe} / \mathrm{H}]=-0.6$ or, more likely, a dynamical origin of this group. Similar to the Hercules stream, this could be either caused by the 6:1 outer Lindblad resonance with the bar at the solar position (B. Fuchs, unpublished) or, as suggested recently by Minchev et al. (2009), the relaxation of disk stars after the perturbation through a massive merger $\sim 1.9$ Gyr ago. Bovey et al. (2009) also suggest a dynamical origin due the very small $\sigma_{V}$ velocity dispersion they find for the Arcturus stream.

The overdensity of stars on slightly retrograde orbits in Fig. $6\left(V_{\text {rot }} \sim-50 \mathrm{~km} \mathrm{~s}^{-1}\right.$ or, equivalently, $V \sim-270 \mathrm{~km} \mathrm{~s}^{-1}$ ) was first noticed by Dinescu (2002), most prominently when the sample was restricted to the metallicity range $-2.0 \leq[\mathrm{Fe} / \mathrm{H}] \leq-1.5$. As these values are consistent with those of the peculiar globular cluster $\omega$ Cen, which is confined to the disk $\left(z_{\max } \sim 1 \mathrm{kpc}\right)$ and has therefore long been suspected to be the surviving nucleus of a tidally disrupted dwarf galaxy (Freeman 1993), Dinescu hypothesized that the peak at $V_{\text {rot }} \sim-50 \mathrm{~km} \mathrm{~s}^{-1}$ was caused by tidal debris from that same galaxy (see also Brook et al. 2004a). Further support for this theory came from Meza et al. (2005), who picked out 13 likely $\omega$ Cen group members by their $L_{z}$ and $|W|$ values from the compilation of $\sim 150$ nearby metal-poor stars from Gratton

\footnotetext{
8 The Williams et al. (2009) selection of Arcturus group members by $V=-100 \pm 10 \mathrm{~km} \mathrm{~s}^{-1}$ differs slightly from the definition of the stream in other works: $\langle V\rangle=-110 \mathrm{~km} \mathrm{~s}^{-1}$ (Eggen 1996); $\langle V\rangle=-120 \mathrm{~km} \mathrm{~s}^{-1}$ (Navarro et al. 2004); $\langle V\rangle=-125 \mathrm{~km} \mathrm{~s}^{-1}$ (Arifyanto and Fuchs 2006); $\langle V\rangle=-105 \mathrm{~km} \mathrm{~s}^{-1}$ (Bovey et al. 2009). Note that such offsets could arise through different values for the LSR.
} 
et al. (2003). The broad ( $-300 \mathrm{~km} \mathrm{~s}^{-1} \lesssim U \lesssim 300 \mathrm{~km} \mathrm{~s}^{-1}$ ), symmetric and centrally under-represented $U$-distribution of these stars indicates that most of them currently move away and towards their apocenter, with only two stars having $|U|<50 \mathrm{~km} \mathrm{~s}^{-1}$. This agrees well with the apocenter of $\omega$ Cen currently lying inside the solar circle at $\sim 6$ kpc. Apart from the two kinematic outliers, all stars additionally define a narrow track in the $([\alpha / \mathrm{Fe}],[\mathrm{Fe} / \mathrm{H}])$-plane,consistent with the chemical composition of $\omega$ Cen itself and indicative of a protracted phase of star formation in a self-enriching progenitor system. With $\sim 7 \%$, the $\omega$ Cen group contributes to the local halo comparable to the H99 stream (Meza et al. 2005).

$\omega$ Cen 's kinematics also put it in the retrograde region occupied by the Kapteyn moving group, named so by O. J. Eggen after Kaptey's star which lags the LSR with $\approx 290 \mathrm{~km} \mathrm{~s}^{-1}$ (Table 11 in Eggen 1996 and references therein). Recently, 16 members of this stream listed by Eggen have been observed spectroscopically to test the hypothesis that Kapteyn's group in fact originates from the same progenitor as $\omega$ Cen (Wylie-de Boer et al. 2010). At least 14 stars have been shown to be kinematically and chemically ( $[\alpha / \mathrm{Fe}]$ plus $[\mathrm{Cu} / \mathrm{Fe}]$ for 5 stars $)$ consistent with $\omega$ Cen 's stellar populations, but due to their higher energies unlikely to stem from the globular cluster itself. Instead, the possible division of the stars into three $L_{z}$ bands made Wylie-de Boer et al. (2010) hypothesize that these stars were stripped from $\omega$ Cen 's parent galaxy at different orbital passages. The progenitor itself would have had a luminosity of $M_{V} \sim-11$ or $2 \times 10^{6} L_{\odot}$ and a total mass of a few $10^{8} M_{\odot}$. Interestingly, Wylie-de Boer et al. (2010) estimated that such a satellite galaxy is expected to shed several hundred more stars into the solar neighborhood that have yet to be detected. In this regard the retrograde streams found recently by various authors (Dettbarn et al. 2007; Klement et al. 2009, see also Table 1) could add further evidence for tidal debris from $\omega$ Cen 's progenitor system.

\subsubsection{Further halo substructure in the solar neighborhood}

Besides the $\mathrm{H} 99$ and $\omega$ Cen streams, which seem to dominate the fraction of nearby halo stars attributed to tidal debris, the recent years have added several candidates for further halo streams thanks to the increasing number of large samples like SDSS/SEGUE. Arifyanto and Fuchs (2006) noted an overdensity of stars with thick disk kinematics rotating approximately $20 \mathrm{~km} \mathrm{~s}^{-1}$ faster than the Arcturus group, but with very similar metallicity and age (inferred from theoretical isochrones). They confirmed this detection in the Geneva-Copenhagen catalog of solar neighborhood stars. Interestingly, this catalog was also analyzed by Helmi et al. (2006), who noted an overdensity of stars in the APL space in an eccentricity range of $0.3 \leq e<0.5$, with overall kinematics overlapping with the detections of Arcturus and the new stream found by Arifyanto and Fuchs (2006). Helmi et al. further decomposed the stars in this eccentricity range into three groups, based on the trend of their metallicity to decrease with increasing eccentricity in a discontinous fashion. Based on the metallicity distributions, they concluded that the three groups may well be tidal debris from three different progenitors with initial masses of $\sim 4 \times 10^{8} \mathrm{M}_{\odot}$. It should be noted that the $(U, V)$-distributions of these stars indeed show the opposite trend of what would be expected if they had a dynamical (resonant) origin. 
Table 1 Current census of solar neighborhood halo streams

\begin{tabular}{|c|c|c|c|c|c|c|}
\hline Stream & $\begin{array}{l}\left\langle v_{\phi}\right\rangle \\
\left(\mathrm{km} \mathrm{s}^{-1}\right)\end{array}$ & $\begin{array}{l}\sigma_{v_{\phi}} \\
\left(\mathrm{km} \mathrm{s}^{-1}\right)\end{array}$ & $\langle[\mathrm{Fe} / \mathrm{H}]\rangle$ & $\sigma_{[\mathrm{Fe} / \mathrm{H}]}$ & $N$ & References \\
\hline $\mathrm{C} 2$ & -75 & 24 & -1.6 & 0.4 & 53 & Klement et al. (2009) \\
\hline $\mathrm{S}_{3}$ & $-48^{\mathrm{a}}$ & 14 & -1.6 & 0.4 & 33 & Klement et al. (2009) \\
\hline Kapteyn & $-46^{\mathrm{b}}$ & 63 & -1.5 & 0.3 & 14 & Wylie-de Boer et al. (2010) \\
\hline$\omega$ Cen & -30 & - & - & - & 56 & Dinescu (2002) \\
\hline $\mathrm{C} 3$ & -24 & 14 & -1.7 & 0.4 & 44 & Klement et al. (2009) \\
\hline $\mathrm{C} 1$ & -16 & 9 & -1.5 & 0.2 & 32 & Klement et al. (2009) \\
\hline $\mathrm{S}_{3}$ & $-9^{\mathrm{a}}$ & 10 & -1.9 & 0.5 & 10 & Dettbarn et al. (2007) \\
\hline $\mathrm{S}_{2}$ & -7 & 4 & -1.9 & 0.1 & 4 & Dettbarn et al. (2007) \\
\hline $\mathrm{S}_{1}$ & 21 & 2 & -1.7 & 0.5 & 4 & Dettbarn et al. (2007) \\
\hline SKOa & 43 & 25 & -2.0 & 0.2 & 6 & Smith et al. (2009) \\
\hline $\mathrm{R} 2$ & 59 & 15 & -1.4 & 0.3 & 19 & Klement et al. (2009) \\
\hline KFR08 & 69 & 11 & -0.7 & 0.3 & 19 & Bobylev et al. (2010) \\
\hline $\begin{array}{l}\text { Groombridge } \\
1830\end{array}$ & $71^{\mathrm{c}}$ & 18 & - & - & 5 & Eggen and Sandage (1959) \\
\hline RHLS & 99 & 25 & -2.0 & 0.2 & 3 & Re Fiorentin et al. (2005) \\
\hline H99 & 140 & 33 & -1.8 & 0.4 & 33 & $\begin{array}{l}\text { Kepley et al. (2007); } \\
\text { Klement et al. (2009) }\end{array}$ \\
\hline $\mathrm{C} 4$ & 173 & 9 & -2.3 & 0.3 & 20 & Klement et al. (2009) \\
\hline
\end{tabular}

$N$ is the number of total stream members given in the references, $\left\langle v_{\phi}\right\rangle$ and $\langle[\mathrm{Fe} / \mathrm{H}]\rangle$ are the mean rotational velocity and metallicity of the stream, and $\sigma_{v_{\phi}}, \sigma_{[\mathrm{Fe} / \mathrm{H}]}$ the corresponding standard deviations

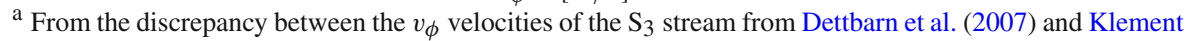
et al. (2009) it follows that both authors might have described two different, but maybe related streams

b The $V$ values adopted by Wylie-de Boer et al. (2010) are based on modern parallaxes, proper motions and radial velocities and differ from the more tightly confined $V$ values originally given in Eggen (1996) ${ }^{\mathrm{c}}$ Eggen and Sandage (1959) used $V_{\odot}=17 \mathrm{~km} \mathrm{~s}^{-1}$, while I applied $5.2 \mathrm{~km} \mathrm{~s}^{-1}$. Note that this group has not yet been confirmed by later studies

The model of Minchev et al. (2009), where the streams found by Arifyanto and Fuchs result from the relaxation of a perturbed disk following a major merger, is also able to explain the location of the moving group detected by Klement et al. (2008) in the first RAVE public data release (Steinmetz et al. 2006), which is at $V \approx-160 \mathrm{~km} \mathrm{~s}^{-1}$. The original detection of the stream in RAVE data was based on the assumption that the vast majority of RAVE stars are dwarfs/ subdwarfs in order to estimate distances through the photometric parallax method. This assumption was shown to be doubtful; in fact, Seabroke et al. (2008) derived from reduced proper motions that $\sim 44 \%$ of RAVE stars are likely K-M giants. The now available second data release (Zwitter et al. 2008), which contains spectroscopically derived $\log g$ estimates for $\sim 21,000$ stars, in principal allows the classification of dwarfs and (sub-)giants and a critical re-analysis of a pure dwarf sample (Klement et al. 2010, in preparation). However, evidence for the existence of the KFR08 stream was found by Klement et al. (2009) in their sample of SEGUE subdwarfs at a confidence level of 99.7\%. Furthermore, Bobylev et al. (2010) identified 19 candidates of the KFR08 stream among a sample of F and 
$\mathrm{G}$ stars for which accurate distance estimates $\left(\sigma_{\pi} / \pi<0.15\right)$ were available from the updated Hipparcos catalog (van Leeuwen 2007); they derived a stream age of 13 Gyr from stellar isochrones and a mean metalliciy of $\langle[\mathrm{Fe} / \mathrm{H}]\rangle=-0.7$ with standard deviation 0.3 dex from Strömgren $u v b y \beta$ photometry. The chemical and chronological homogeinity, as well as the banana-shaped $(U, V)$-distribution of the stars (Fig. 8 in Bobylev et al. 2010), favor a tidal rather than a dynamic origin of the KFR08 stream.

Other moving group candidates without known progenitor systems have been identified by Dettbarn et al. (2007) and Klement et al. (2009) in the space of effective integrals of motion $\left(v, V_{\mathrm{az}}, V_{\Delta \mathrm{E}}\right)$ (Sect. 3.1). They are listed in Table 1 which will be described in the next section. One of the features detected by Dettbarn et al., their ' $\mathrm{S}_{3}$ ' overdensity, was confirmed by Klement et al. and affiliated to a much larger group of stars that also show a spatial coherence with similarity to an overdensity detected by Juric et al. (2008) at $(R, z) \approx(9.5,0.8) \mathrm{kpc}$. The stream members move on slightly retrograde orbits, are currently near their apocenters and have a $[\mathrm{Fe} / \mathrm{H}]$-distribution characterized through $\langle\mathrm{Fe} / \mathrm{H}]\rangle \approx-1.6, \sigma_{[\mathrm{Fe} / \mathrm{H}]}=0.4$. Although this points towards a possible relation with $\omega$ Cen, the high $W$ velocity components and spatial concentration of the stars argue against this hypotheses. A detailed chemical study would be helpful in solving this issue.

\subsection{A census on the currently known halo streams}

In Table 1, I summarize all currently identified halo streams within $2 \mathrm{kpc}$ from the sun, ordered by the $v_{\phi}$ velocity components or, equivalently, their $L_{z}$ values. $v_{\phi}$ is the rotational velocity in a cylindrical coordinate system and for nearby stars can be approximated by $v_{\phi} \approx V+V_{\mathrm{LSR}}+V_{\odot}$. I kept the names originally given to the individual streams by the authors cited at the end of each row. I have omitted to list Arcturus and the AF06 stream (Arifyanto and Fuchs 2006), because their kinematics and chemistry more closely resemble dynamical thick disk streams (Williams et al. 2009).

From Table 1 it is obvious that some of the individual groups might be related in the sense that they might stem from the same progenitor. For example, many of the retrograde streams in the region $-100 \mathrm{~km} \mathrm{~s}^{-1} \lesssim v_{\phi} \lesssim 0$ overlap in their rotational velocity and $[\mathrm{Fe} / \mathrm{H}]$ abundances. As discussed in Sect. 4.1.3, this region is thought to be populated by multiple streams from $\omega$ Cen 's progenitor, and it seems very likely that many of these features are indeed related. In particular, Klement et al. (2009) already showed that the streams ' $\mathrm{C} 2$ ', ' $\mathrm{C} 3$ ' and ' $\mathrm{S}_{3}$ ' are not only kinematically and chemically connected, but also spatially coherent, similar to a small overdensity at $(R, z) \approx(9.5,0.8) \mathrm{kpc}$ described by Juric et al. (2008). Other streams with a common origin might be the RHLS and H99 groups (Re Fiorentin et al. 2005), R2 and KFR08 (Klement et al. 2009) and the $S_{3}$ streams from Dettbarn et al. (2007) and Klement et al. (2009), respectively.

Furthermore, relations between halo streams and certain globular clusters have been suggested in several instances, based on their common position in the IoM-space. In particular, relations have been suggested between SKOa and the clusters NGC 5466, NGC 6934, NGC 7089/M2 and NGC 6205/M13 (Smith et al. 2009). Also, as already 
discussed in Sect. 4.1.3, Kapteyn's stream has been linked to $\omega$ Cen, and probably the globular clusters NGC 362 and NGC 6779 are associated, too (Dinescu 2002).

To draw further conclusions about the origins of, and relations between, the several streams one would need (i) more precise 3D velocity estimates $\left(\lesssim 5 \mathrm{~km} \mathrm{~s}^{-1}\right.$ Helmi and White 1999), which will ultimately become available for thousands of halo stars from the anticipated Gaia mission and (ii) detailed abundance analyses of the individual streams like the ones undertaken by Wylie-de Boer et al. (2010) and Roederer et al. (2010).

\section{Conclusions}

In this article, my goal was to summarize the current state of research on stellar halo streams in the solar neighborhood. I gave an overview on the processes that play a role in the disruption of Milky Way satellites and the subsequent formation of stellar streams that eventually pass the vicinity of the sun. Disentangling and correlating the individual streams that contribute to the local halo thus gives valuable insights into the history of the Milky Way in the context of hierarchical galaxy formation. Various search strategies in complementary data sets should be used for this task in order to increase the confidence levels on the existence of individual streams. Further, spectroscopic studies of the various streams in the style of Williams et al. (2009), Wylie-de Boer et al. (2010) or Roederer et al. (2010) are necessary to infer clues about their origin (dynamical vs. accreted), properties of any progenitor system and eventual relationships between individual streams. To fully resolve the hundreds of expected individual halo streams, 3D velocities out to at least $2 \mathrm{kpc}$ have to be measured with accuracies better than $5 \mathrm{~km} \mathrm{~s}^{-1}$. In addition, estimating their time since accretion requires precise measurements of parallaxes good to 1-2\% (Gómez and Helmi 2010b). Ultimately, Gaia will deliver these informations, but—as this review hopefully demonstrated —in the meantime, many discoveries and conclusions are already possible with currently available data and facilities.

Acknowledgements I would like to thank the following people: Burkhard Fuchs, Chao Liu and Amina Helmi for useful comments on an early draft of this article; Christian Dettbarn for kindly providing me the parameters of the individual stars from his article; Amina Helmi for allowing me to use the data of her simulations in Figs. 2 and 4; Facundo A. Gomez and Andres Meza for providing me with high-resolution figures from their articles. I acknowledge financial support from Deutsches Zentrum für Luft- und Raumfahrt.

Open Access This article is distributed under the terms of the Creative Commons Attribution Noncommercial License which permits any noncommercial use, distribution, and reproduction in any medium, provided the original author(s) and source are credited.

\section{References}

Abadi MG, Navarro JF, Steinmetz M, Eke VR (2003) Simulations of galaxy formation in a $\Lambda$ cold dark matter universe. I. Dynamical and photometric properties of a simulated disk galaxy. Astrophys J 591:499-514

Abadi MG, Navarro JF, Steinmetz M, Eke VR (2003) Simulations of galaxy formation in a $\Lambda$ cold dark matter universe. II. The fine structure of simulated galactic disks. Astrophys J 597:21-34 
Allende Prieto C, Sivarani T, Beers TC, Lee YS, Koesterke L et al (2008) The SEGUE stellar parameter pipeline. III. Comparison with high-resolution spectroscopy of SDSS/SEGUE field stars. Astrophys J 136:2070-2082

Antoja T, Figueras F, Fernández D, Torra J (2008) Origin and evolution of moving groups. I. Characterization in the observational kinematic-age-metallicity space. Astron Astrophys 490:135-150

Arifyanto MI, Fuchs B (2006) Fine structure in the phase space distribution of nearby subdwarfs. Astron Astrophys 499:533-538

Arnold R, Gilmore G (1992) Halo blue horizontal branch stars-spectroscopy in two fields. Mon Not R Astron Soc 257:225-239

Beers TC, Chiba M, Yoshiii Y, Platais I, Hanson RB, Fuchs B, Rossi S (2000) Kinematics of metal-poor stars in the galaxy. II. Proper motions for a large nonkinematically selected sample. Astron J 119:2866-2881

Bekki K, Chiba M (2001) Formation of the galactic stellar halo. I. Structure and kinematics. Astrophys J 558:666-686

Belokurov V, Zucker DB, Evans NW, Gilmore G, Vidrih S et al (2006) The field of streams: Sagittarius and its siblings. Astrophys J Lett 642:L137-L140

Belokurov V, Evans NW, Irwin MJ, Hewett PC, Wilkinson MI (2006) The discovery of tidal tails around the globular cluster NGC 5466. Astrophys J Lett 637:L29-L32

Binney J, Spergel D (1984) Spectral stellar dynamics. II The action integrals. Mon Not R Astron Soc 206:159-177

Binney J, Tremaine S (2008) Galactic dynamics, 2nd edn. Princeton University Press, Princeton

Bobylev VV, Bajkova AT, Mylläri AA (2010) Analysis of peculiarities of the stellar velocity field in the solar neighborhood. Astron Lett 36:27-43

Bovy J, Hogg DW, Roweis ST (2009) The velocity distribution of nearby stars from Hipparcos data. I. The significance of the moving groups. Astrophys J 700:1794-1819

Boylan-Kolchin M, Ma CP, Quataert E (2008) Dynamical friction and galaxy merging time-scales. Mon Not R Astron Soc 383:93-101

Brook CB, Kawata D, Gibson BK (2004a) Phase space plots of streams in the halo and their chemical tags. In: Prada F, Martinez Delgado D, Mahoney TJ (eds) Phase space plots of streams in the halo and their chemical tags, ASPC 327, pp 100-103

Brook CB, Kawata D, Gibson BK, Freeman KC (2004b) The emergence of the thick disk in a cold dark matter universe. Astrophys J 612:894-899

Bullock JS, Johnston KV (2005) Tracing galaxy formation with stellar halos. I. Methods. Astrophys J 635:931-949

Carollo D, Beers TC, Lee YS, Chiba M, Norris JE, Wilhelm R, Sivarani T, Marsteller B, Munn JA, BailerJones CAL, Fiorentin PR, York DG (2007) Two stellar components in the halo of the Milky Way. Nature 450:1020-1025

Chandrasekhar S (1943) Dynamical friction. I. General considerations: the coefficient of dynamical friction. Astrophys J 97:255-262

Chiba M, Beers TC (2000) Kinematics of metal-poor stars in the galaxy. III. Formation of the stellar halo and thick disk as revealed from a large sample of nonkinematically selected stars. Astron J 119:2843-2865

Choi JW, Weinberg MD, Katz N (2007) The dynamics of tidal tails from massive satellites. Mon Not R Astron Soc 381:987-1000

Cole S, Aragon-Salamanca A, Frenk CS, Navarro JF, Zepf SE (1994) A recipe for galaxy formation. Mon Not R Astron Soc 271:781-806

Colpi M, Mayer L, Governato F (1999) Dynamical friction and the evolution of satellites in virialized halos: the theory of linear response. Astrophys J 525:720-733

Cooper AP, Cole S, Frenk CS, White SDM, Helly J et al (2010) Mon Not R Astron Soc 406:744-766

De Silva GM, Freeman KC, Bland-Hawthorn J, Asplund M, Bessel MS (2007) Chemically tagging the HR 1614 Moving Group. Astron J 133:694-704

De Simone R, Wu X, Tremaine S (2004) The stellar velocity distribution in the solar neighbourhood. Mon Not R Astron Soc 350:627-643

Dehnen W (1998) The distribution of nearby stars in velocity space inferred from HIPPARCOS data. Astron J 115:2384-2396

Dehnen W (2000) The effect of the outer lindblad resonance of the galactic bar on the local stellar velocity distribution. Astron J 119:800-812

Dekker E (1976) Spiral structure and the dynamics of galaxies. Phys Rep 24:315-389 
Dettbarn C, Fuchs B, Flynn C, Williams M (2007) Signatures of star streams in the phase space distribution of nearby halo stars. Astron Astrophys 474:857-861

Dinescu DI (2002) A solar neighborhood search for tidal debris from $\omega$ Centauri's hypothetical parent galaxy. In: van Leeuwen F, Hughes JD, Piotto G (eds) Omega Centauri, a unique window into astrophysics. ASPC 265, pp 365-379

Doinidis SP, Beers TC (1989) Astrophys J Lett 340:L57-L60

Eggen OJ (1958) Stellar groups. I. The Hyades and Sirius groups. Mon Not R Astron Soc 118:65-79

Eggen OJ, Sandage A (1959) Stellar groups, IV. The Groombridge 1830 group of high velocity stars and its relation to the globular clusters. Mon Not R Astron Soc 119:255-277

Eggen OJ, Lynden-Bell D, Sandage AR (1962) Evidence from the motions of old stars that the galaxy collapsed. Astrophys J 136:748-767

Eggen OJ (1971) The Arcturus group. Publ Astron Soc Pac 83:271-285

Eggen OJ (1977) Intermediate band photometry of late-type stars. II-Some stellar groups. Astrophys J 215:812-826

Eggen OJ (1996) Star streams and galactic structure. Astron J 112:1595-1613

Fellhauer M, Evans NW, Belokurov V, Zucker DB, Yanny B, Wilkinson MI, Gilmore G, Irwin MJ, Bramich DM, Vidrih S, Hewett P, Beers T (2007) Is Ursa Major II the progenitor of the orphan stream. Mon Not R Astron Soc 375:1171-1179

Finlator K, Ivezić Ž, Fan X, Strauss MA, Knapp GR, Lupton RH, Gunn JE, Rockosi CM, Anderson JE, Csabai I, Hennessy GS, Hindsley RB, McKay TA, Nichol RC, Schneider DP, Smith JA, York DG and the SDSS Collaboration (2000) Optical and infrared colors of stars observed by the two micron all sky survey and the sloan digital sky survey. Astron J 120:2615-2626

Font AS, Johnston KV, Bullock JS, Robertson BE (2006) Chemical abundance distributions of galactic halos and their satellite systems in a $\Lambda C D M$ universe. Astrophys J 638:585-595

Freeman KC (1993) Globular clusters and nucleated dwarf ellipticals. In: Smith GH, Brodie JP (eds) The globular cluster-galaxy connection. ASPC 48, pp 608-614

Fujii M, Funato Y, Makino J (2006) Dynamical friction on satellite galaxies. Publ Astron Soc Jpn 58:743752

Gill SPD, Knebe A, Gibson BK, Dopita MA (2004) The evolution of substructure-II. Linking dynamics to environment. Mon Not R Astron Soc 351:410-422

Gómez FA, Helmi A (2010) On the identification of substructure in phase space using orbital frequencies. Mon Not R Astron Soc 401:2285-2298

Gómez FA, Helmi A (2010) On the identification of merger debris in the Gaia Era. arXiv:1004.4974v1

Gould A (2003) An upper limit on the granularity of the local stellar halo. Astrophys J Lett 592: L63-L66

Governato F, Willman B, Mayer L, Brooks A, Stinson G et al (2007) Forming disc galaxies in $\lambda$ CDM simulations. Mon Not R Astron Soc 374:1479-1494

Gratton RG, Carretta E, Claudi R, Lucatello S, Barbieri M (2003) Abundances for metal-poor stars with accurate parallaxes. I. Basic data. Astron Astrophys 404:187-210

Grillmair CJ, Johnson R (2006) The detection of a $45^{\circ}$ tidal stream associated with the globular cluster NGC 5466. Astrophys J Lett 639:L17-L20

Grillmair CJ, Dionatos O (2006) A $22^{\circ}$ tidal tail for Palomar 5. Astrophys J Lett 641:L37-L39

Hashimoto Y, Funato Y, Makino J (2003) To circularize or not to circularize? Orbital evolution of satellite galaxies. Astrophys J 582:196

Haywood M (2008) Radial mixing and the transition between the thick and thin galactic discs. Mon Not R Astron Soc 388:1175-1184

Helmi A, White SDM, de Zeeuw PT, Zhao H (1999) Debris streams in the solar neighbourhood as relicts from the formation of the Milky Way. Nature 402:53-55

Helmi A, White SDM (1999) Building up the stellar halo of the galaxy. Mon Not R Astron Soc 307: 495-517

Helmi A, de Zeeuw PT (2000) Mapping the substructure in the galactic halo with the next generation of astrometric satellites. Mon Not R Astron Soc 319:657-665

Helmi A, White SDM, Springel V (2003) The phase-space structure of cold dark matter haloes: insights into the galactic halo. Mon Not R Astron Soc 339:834-848

Helmi A, Navarro JF, Nordström B, Holmberg J, Abadi MG, Steinmetz M (2006) Pieces of the puzzle: ancient substructure in the galactic disc. Mon Not R Astron Soc 365:1309-1323

Ibata R, Gilmore G, Irwin MJ (1994) A dwarf satellite galaxy in Sagittarius. Nature 370:194-196 
Ibata R, Irwin M, Lewis GF, Stolte A (2001) Galactic halo substructure in the sloan digital sky survey: the ancient tidal stream from the sagittarius dwarf galaxy. Astrophys J Lett 547:L133-L136

Johnston KV (1998) A prescription for building the Milky Way's halo from disrupted satellites. Astrophys J 495:297-308

Juric M, Ivezić Ž, Brooks A, Lupton RH, Schlegel D et al (2008) The Milky Way tomography with SDSS. I. Stellar number density distribution. Astrophys J 673:864-914

Katz N (1992) Dissipational galaxy formation. II-Effects of star formation. Astrophys J 391:502-517

Kazantzidis S, Bullock JS, Zentner AR, Kravtsov AV, Moustakas LA (2008) Cold dark matter substructure and galactic disks. I. Morphological signatures of hierarchical satellite accretion. Astrophys J 688:254-276

Kepley AA, Morrison HL, Helmi A, Kinman TD, van Duyne J, Martin JC, Harding P, Norris JE, Freeman KC (2007) Halo star streams in the solar neighborhood. Astron J 134:1579-1595

Klement R, Fuchs B, Rix HW (2008) Identifying stellar streams in the first RAVE public data release. Astrophys J 685:261-271

Klement R, Rix HW, Flynn C, Fuchs B, Beers TC et al (2009) Halo streams in the seventh sloan digital sky survey data release. Astrophys J 698:865-894

Li YS, Helmi A (2008) Infall of substructures on to a Milky Way-like dark halo. Mon Not R Astron Soc 385:1365-1373

Majewski SR (1993) The Milky-Way—clues to a merger past. In: Majewski SR (ed) Galaxy evolution. The Milky Way perspective. Astronomical Society of the Pacific Conference Series 49:5-24

Majewski SR, Munn JA, Hawley SL (1996) Absolute proper motions to B approximately 22.5: Large-scale streaming motions and the structure and origin of the galactic halo. Astrophys J Lett 495:L73-L77

Majewski SR, Skrutskie MF, Weinberg MD, Ostheimer JC (2003) A two micron all sky survey view of the Sagittarius dwarf galaxy. I. Morphology of the Sagittarius core and tidal arms. Astrophys J 599:10821115

McGill C, Binney J (1990) Torus construction in general gravitational potentials. Mon Not R Astron Soc 244:634-645

McMillan PJ, Binney JJ (2008) Disassembling the Galaxy with angle-action coordinates. Mon Not R Astron Soc 390:429-437

Meza A, Navarro JF, Abadi MG, Steinmetz M (2005) Accretion relics in the solar neighbourhood: debris from $\omega$ Cen's parent galaxy. Mon Not R Astron Soc 359:93-103

Minchev I, Quillen AC, Williams M, Freeman KC, Nordhaus J, Siebert A, Bienaymé O (2009) Is the Milky Way ringing? The hunt for high-velocity streams. Mon Not R Astron Soc 396:L56-L60

Mo HJ, Mao S, White SDM (1998) The formation of galactic discs. Mon Not R Astron Soc 295:319-336

Morrison HL, Helmi A, Sun J, Liu P, Gu R, Norris JE, Harding P, Kinman TD, Kepley AA, Freeman KC, Williams M, Van Duyne J (2009) Fashionably late? Building up the Milky Way'S inner halo. Astrophys J 694:130-143

Navarro JF, Helmi A, Freeman KC (2004) The extragalactic origin of the Arcturus group. Astrophys J 601:L43-L46

Newberg HJ, Yanny B, Rockosi C, Grebel EK, Rix HW et al (2002) The ghost of Sagittarius and lumps in the halo of the Milky Way. Astrophys J 569:245-274

Nissen PE, Schuster WJ (2010) Two distinct halo populations in the solar neighborhood. Evidence from stellar abundance ratios and kinematics. Astron Astrophys 511:L10-L4

Nordström B, Mayor M, Andersen J, Holmberg J, Pont F, Jorgensen BR, Olsen EH, Udry S, Mowlavi N (2004) The Geneva-Copenhagen survey of the solar neighbourhood. Astron Astrophys 418:989_ 1019

Odenkirchen M, Grebel EK, Rockosi CM, Dehnen W, Ibata R et al (2001) Detection of Massive Tidal Tails around the Globular Cluster Palomar 5 with Sloan Digital Sky Survey Commissioning Data. Astrophys J Lett 548:L165-L169

Ollongren A (1962) Three-dimensional galactic stellar orbits. Bull Astron Inst Neth 16: 241-296

Oort JH (1965) Stellar dynamics. In: Blaauw A, Schmidt M (eds) Galactic structure. University of Chicago Press, Chicago pp 455-512

Peñarrubia J, Benson AJ, Martínez-Delgado D, Rix HW (2006) Modeling tidal streams in evolving dark matter halos. Astrophys J 645:240-255

Perryman MAC, de Boer KS, Gilmore G, Høg E, Lattanzi MG, Lindegren L, Luri X, Mignard F, Pace O, de Zeeuw PT (2001) GAIA: composition, formation and evolution of the galaxy. Astron Astrophys 369:339-363 
Poveda A, Allen C, Schuster W (1992) Moving clusters among galactic halo stars. In: Barbuy B, Renzini A (eds) The stellar populations of galaxies. IAU Symposium 149, Kluwer Academic Publishers, Dordrecht, p 471

Press WH, Schechter P (1974) Formation of galaxies and clusters of galaxies by self-similar gravitational condensation. Astrophys J 187:425-438

Proctor RA (1869) Preliminary paper on certain drifting motions of the stars. R Soc Lond Proc I 18:169-171

Re Fiorentin P, Helmi A, Lattanzi MG, Spagna A (2005) Structure in the motions of the fastest halo stars. Astron Astrophys 439:551-558

Read JI, Lake G, Agertz O, Debattista VP (2008) Thin, thick and dark discs in $\Lambda$ CDM. Mon Not R Astron Soc 389:1041-1057

Roederer IU, Sneden C, Thompson IB, Preston GW, Shectman SA (2010) Characterizing the chemistry of the Milky Way stellar halo: detailed chemical analysis of a metal-poor stellar stream. Astrophys J 711:573-596

Sales LV, Navarro JF, Abadi MG, Steinmetz M (2007) Satellites of simulated galaxies: survival, merging and their relationto the dark and stellar haloes. Mon Not R Astron Soc 379:1464-1474

Sales LV, Helmi A, Abadi MG, Brook CB, Gómez FA, Roskar R, Debattista VP, House E, Steinmetz M, Villalobos Á (2009) Orbital eccentricity as a probe of thick disk formation scenarios. Mon Not R Astron Soc 400:L61-L65

Samland M (2004) A model for the formation of the Milky Way. PASA 21:175-178

Schönrich R, Binney J (2009) Origin and structure of the galactic disc(s). Mon Not R Astron Soc 399:11451156

Seabroke GM, Gilmore G, Siebert A, Bienaymé O, Binney J et al (2008) Is the sky falling? Searching for stellar streams in the local Milky Way disc in the CORAVEL and RAVE surveys. Mon Not R Astron Soc 384:11-32

Searle L, Zinn R (1978) Compositions of halo clusters and the formation of the galactic halo. Astrophys J 225:357-379

Sellwood JA, Binney JJ (2002) Radial mixing in galactic discs. Mon Not R Astron Soc 336:785-796

Skrutskie MF, Cutri RM, Stiening R, Weinberg MD, Schneider S, et al (2006) The two micron all sky survey (2MASS). Astron J 131:1163

Smith MC, Evans NW, Belokurov V, Hewett PC, Bramich DM, Gilmore G, Irwin MJ, Vidrih S, Zucker DB (2009) Kinematics of SDSS subdwarfs: structure and substructure of the Milky Way halo. Mon Not R Astron Soc 399:1223-1237

Steinmetz M, Navarro JF (1999) The cosmological origin of the Tully-Fisher relation. Astrophys J 513:555560

Steinmetz M, Navarro JF (2002) The hierarchical origin of galaxy morphologies. New Astron 7:155-160

Steinmetz M, Zwitter T, Siebert A, Watson FG, Freeman KC et al (2006) The radial velocity experiment (RAVE): first data release. Astron J 132:1645-1668

Stoughton C, Lupton RH, Bernardi M, Blanton MR, Burles S et al (2002) Sloan digital sky survey: early data release. Astron J 123:485-548

Tremaine S (1993) A pessimist's view of galactic structure. In: Holt SS, Verter F (eds) Back to the galaxy. AIP conference proceedings 278, New York, pp 599-609

Tremaine S (1999) The geometry of phase mixing. Mon Not R Astron Soc 307:877-883

van Leeuwen F (2007) Validation of the new Hipparcos reduction. Astron Astrophys 474:653-664

Venn KA, Irwin M, Shetrone MD, Tout CA, Hill V, Tolstoy E (2004) Stellar chemical signatures and hierarchical Galaxy formation. Astrophys J 128:1177-1195

Villalobos A, Helmi A (2008) Simulations of minor mergers-I. General properties of thick discs. Mon Not R Astron Soc 391:1806-1827

Villalobos A, Helmi A (2009) Simulations of minor mergers-II. The phase-space structure of thick discs. Mon Not R Astron Soc 399:166-176

Vivas AK, Zinn R, Andrews P, Bailyn C, Baltay C et al (2001) The QUEST RR Lyrae survey: confirmation of the clump at 50 kiloparsecs and other overdensities in the outer halo. Astrophys J Lett 554:L33-L36

Vogelsberger M, White SDM, Helmi A, Springel V (2008) The fine-grained phase-space structure of cold dark matter haloes. Mon Not R Astron Soc 385:236-254

Warnick K, Knebe A, Power C (2008) The tidal streams of disrupting subhaloes in cosmological dark matter haloes. Mon Not R Astron Soc 385:1859-1883

White SDM (1976) A note on the minimum impact parameter for dynamical friction involving spherical clusters. Mon Not R Astron Soc 174:467-470 
White SDM, Rees MJ (1978) Core condensation in heavy halos-a two-stage theory for galaxy formation and clustering. Mon Not R Astron Soc 183:341-358

Williams MEK, Freeman KC, Helmi A and the RAVE collaboration (2009) The arcturus moving group: its place in the galaxy. In: Andersen J, Bland-Hawthorn J, Nordström B (eds) The galaxy disk in cosmological context. IAU Symposium 254, pp 139-144

Wylie-de Boer EC, Freeman KC, Williams M (2010) Evidence of tidal debris from $\omega$ Cen in the Kapteyn group. Astron J 139:636-645

Yanny B, Rockosi C, Newberg HJ, Knapp GR, Adelman-McCarthy JK et al (2009) SEGUE: a spectroscopic survey of 240,000 stars with $g=14-20$. Astron J 137:4377-4399

York DG, Adelman J, Anderson JE Jr, Anderson SF, Annis J et al (2000) The sloan digital sky survey: technical summary. Astron J 120:1579-1587

Zhao H (2004) Dynamical friction for dark halo satellites: effects of tidal mass loss and growing host potential. Mon Not R Astron Soc 351:891-902

Zwitter T, Siebert A, Munari U, Freeman KC, Siviero A et al (2008) The radial velocity experiment (RAVE): second data release. Astron J 136:421-451 